\title{
Atomic-scale investigation of triple junction role on defects binding energetics and structural stability in $\alpha-F e$
}

\author{
I. Adlakha, and K.N. Solanki* \\ School for Engineering of Matter, Transport, and Energy, Arizona State University, Tempe, AZ \\ *(480)965-1869; (480)727-9321 (fax), E-mail: kiran.solanki@asu.edu, (Corresponding author)
}

\begin{abstract}
Nanocrystalline (NC) metals (mean grain sizes $\mathrm{d} \leq 100 \mathrm{~nm}$ ) have enhanced mechanical strength as compared to coarse-grained metals $(\mathrm{d} \geq 1 \mu \mathrm{m})$, and thus, are a promising alternative as structural materials for future high energy nuclear reactors. However, during extreme conditions, the NC microstructure has been found to be thermodynamically unstable, thereby limiting its applicability. Further, for materials with average grain size $<10 \mathrm{~nm}$, the triple junctions (TJs) have been observed to have a significant contribution on the mechanical behavior and microstructural stability. Moreover, at the atomic-scale, the region surrounding the TJ demonstrates unique physical properties, such as rapid diffusion, non-equilibrium segregation and increased dislocation activity. Therefore, in this work, we systematically assess the role of TJs on the structural stability and the solute binding behavior in $\alpha$-Fe. Using atomistic simulations, we show that the TJ resolved line tension is strongly correlated (inversely) with the mean activation energy for self-diffusion along the TJ, i.e., the thermodynamically unstable TJs demonstrated a lower activation energy barrier for self-diffusion along TJs with higher line tension. Next, we demonstrated that the strain energy evolution around the TJ can provide insights into the distinct binding behavior of point defects and solute atoms. In other words, the examination of solute binding behavior revealed a localized region of stable sites around the TJs which aids in accommodation of high solute concentration at high temperatures. In summary, our findings quantify the distinct role of TJs on the defect (vacancy, self-interstitial and solute atom) binding and migration behavior and these findings are necessary for designing future structural materials for extreme environments, including those needed in aerospace, naval, civilian and energy sector infrastructures.
\end{abstract}

Keywords: Nanocrystalline; Triple junctions; Grain boundaries; Solutes 


\section{Introduction}

The next generation of nuclear reactors requires structural materials that can withstand the combined effects of high radiation dosage, elevated temperature and corrosive environment for an extended period of time. These demands necessitate the development of new avenues in material design/processing technology [1-3]. For example, the continuous production of He as a result of the transmutation reactions $(\eta, \alpha)$ increases the susceptibility of He bubble formation along the grain boundaries (GBs) thereby degrading the material strength. Thus, tailoring microstructural features such as grain or cell boundaries along with triple junctions (TJs) and free surfaces which act as sinks for the radiation induced defects (point defects, solute-point defect clusters) [4,5] is critical in development of new structural alloys for nuclear applications. Recent development of advanced materials such as nanoporous [6,7], nanocrystalline (NC) [8-12], and nanocomposite materials $[13,14]$ show promise for use as structural materials in extreme environments $[9,10]$. However, the grand challenge with broader applicability of such nanostructured materials has been the stability of the non-equilibrium microstructure during processing and deformation [15-19]. This significant degradation in properties of nanostructured materials is in part due to an increase in the large volume fraction of GBs along with TJs, which lack long-range crystalline order leading to diffusional processes such as sliding and/or rotation [20]. Further, in nanostructured materials, such as NC metals, as the mean grain size decreases below $10 \mathrm{~nm}$, the percent microstructure constituted by GBs and TJs increases and can be in excess of $50 \%$ [21,22]. Thus, due to the large volume fractions of non-equilibrium defects, NC metals exhibit altered physical responses to deformation, temperature, and radiation [15-19], i.e., the structural stability of NC materials is driven by GBs (planar defects), TJs (line defects), and their underlying structures $[23,21]$. Hence, a fundamental understanding of the relationship between the line/planar defect structures and the associated properties is highly relevant to develop stable, interface-dominant materials. Further, the role of TJ atomic structure on mass transport or diffusional processes, which directly control the stability of NC materials, is a critical open question, especially for nuclear applications $[1,3]$.

Radiation induced segregation can cause void swelling, phase transformation, precipitation, and compositional changes in structural materials [24-26]. Irradiation by high energy particles produces a large quantity of point defects throughout the material, and hence, the migration of these defects towards dislocations, free surfaces, GBs, TJs, and other microstructural sinks 
creates a defect flux [27-29]. Therefore, quantifying how point defects interact with defect sinks, such as GBs/TJs, is also important for understanding the strength of interfaces in high radiation environments. Furthermore, during irradiation, the flux of solute and impurity elements is highly coupled with the flux of vacancies and interstitials giving rise to radiation induced segregation. As point defects diffuse and bind to microstructural sinks, solute and impurity atoms are spatially redistributed in the vicinity of these sinks [30]. The net result is an accumulation or a depletion of elements at these defect sinks, which can have deleterious effects on material properties and stability [31]. Atomistic and electronic simulations are increasingly being utilized as tools for investigating such fundamental mechanisms associated with defect segregation/binding behavior. It has been shown that impurity segregation to GBs can have a profound effect on the mechanical behavior, i.e., a significant beneficial effect [32-34] or a significant detrimental effect [35-40]. For example, Solanki et al. [41] found that segregation of certain $\mathrm{H}$ defects is favored at $\alpha-\mathrm{Fe}$ GBs resulting in a reduction of grain boundary cohesive strength. On the other hand, Yamaguchi [34] has shown that the segregation of boron and carbon to GBs can actually be beneficial by strengthening grain boundary cohesion. These results indicate that the segregation behavior of these elements plays an important role in grain boundary embrittlement or strengthening behavior. Moreover, the TJ and associated GB structures can influence the segregation behavior. Recently, Adlakha and Solanki [22] used atomistic simulations to show how the local TJ structure affects the sink strength for vacancy and self-interstitial point defects in $\mathrm{Al}, \mathrm{Ni}$, and $\mathrm{Cu}$. Yin et al. [42] quantified the solute concentrations near a TJ and found that it far exceeded the concentrations observed along the constituent GBs. Therefore, in the case of NC materials, understanding the behavior of solute atoms and point defects in the vicinity of TJs becomes highly relevant.

In this work, for the first time, we systematically investigate the structural stability of TJs and the solute concentration in $\alpha$-Fe using atomistic simulations along with the nudge elastic band (NEB) method [43]. A range of special TJs that involve the $\Sigma 3$ GB (these TJs have been shown to occur frequently [44-46]) were investigated. Further, the role of the atomic structure surrounding the $\mathrm{TJ}$ on the binding energetics of point defects, (vacancy and self-interstitial atom) and various solute atoms $(\mathrm{H}, \mathrm{He}, \mathrm{P}$, and V) was assessed. The paper is organized as follows. The next section describes the computational methods and GB/TJ generation procedures employed. Then, we discuss the TJ resolved line tension, the TJ strain energy evolution behavior and their 
implications on the structural stability and binding energetics. For instance, we show that the TJ resolved line tension is strongly correlated (inversely) with the mean activation energy for selfdiffusion along the TJ. In other words, the thermodynamically unstable TJs (i.e., comparatively higher resolved line tension) demonstrated a lower energy barrier for self-diffusion along TJs. Further, the TJ strain energy evolution was found to accurately predict the binding behavior of point defects and solute atoms (i.e., beneficial effect or a significant detrimental effect). This, together with the resolved line tension, provides the necessary length-scale parameters for engineering radiation resistant $\mathrm{NC}$ materials with a favorable population of TJs (similar to the GB/interface engineering).

\section{Computational methods}

Molecular dynamics simulations using LAMMPS [47] and MOLDY [48] were employed to investigate the role of TJs on various phenomena that occur under radiation damage. Specifically, various intrinsic properties were quantified to correlate the diffusion kinetics of point defects and the radiation induced segregation behavior of $\mathrm{H}, \mathrm{He}, \mathrm{P}$, and $\mathrm{V}$ atoms in the vicinity of various TJs. Atomistic simulations were performed using semi-empirical embedded atom method (EAM) potentials to accurately describe various Fe-solute interactions [49-52]. In all of these EAM potentials, the Fe-Fe interactions were based on the Fe EAM potential of Mendelev et al. [53] which compares well with Chamati et al. [54]. In the case of Fe-He, a modified version of the MOLDY code was used [55,56]. These EAM potentials were parameterized using an extensive database of energies and configurations from density functional theory calculations and have been used to accurately predict different material properties such as cohesive energy, surface energies, generalized stacking fault energies and solute-defect interactions (for details, see Refs. [41,49,56-60]). The cohesive energy predicted by all the potentials for $\alpha$-Fe was approximately $4.01 \mathrm{eV} /$ atom, which compares well with 4.2 eV/atom reported by Bhatia et al. [61] using density functional theory. 


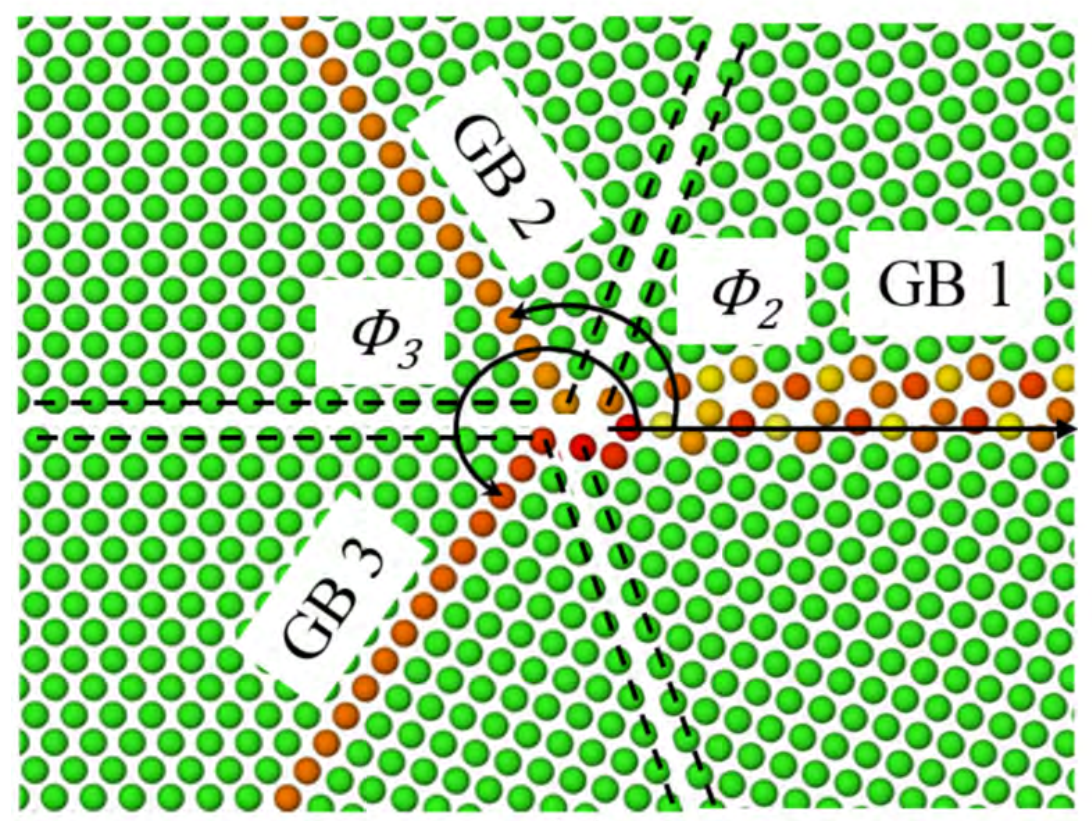

(a)
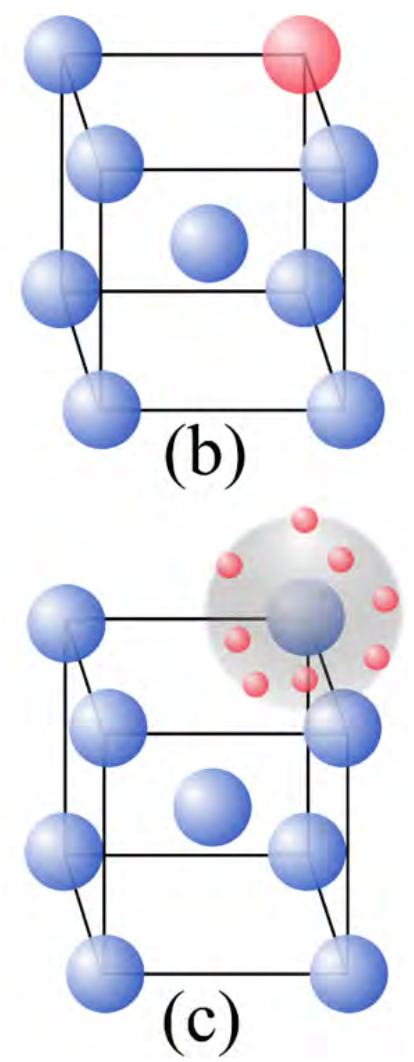

Figure 1: a) Schematic showing construction of a GB TJ configuration. The GBs were minimized separately and a wedge was carved out along the $\{001\} /\{011\}$ planes represented by dashed lines. b) The initial configuration of the substitutional solute atom (red atom) before energy minimization in a $\mathrm{BCC} F e$ (blue atoms) unit cell. c) An example of a starting instantiation of an interstitial solute atom that lies within a radius of $\sqrt{3} / 2 a_{0}$ around the $\alpha$ atomic site.

In this work, the TJs were constructed using $<110>$ symmetric tilt grain boundaries (GBs) (see Table 1). These GBs were characterized using the structural unit method [62,63]. As with past work [41,64-68], an atom deletion criterion, multiple initial configurations and various inplane rigid body translations were utilized to accurately obtain an optimal minimum energy GB structure via the conjugate gradient energy minimization process. Circular wedges were then cut from the GBs along the stitch plane, i.e., $\{001\} /\{011\}$ planes (see Figure 1a) $[22,69,70]$. Next, the GBs labelled 2 and 3 were rotated by $\phi_{2}$ and $\phi_{3}$ respectively about the GB tilt axis, as shown in Figure 1a. The three circular wedges were brought together, the overlapping atoms were removed, and the energy minimization was carried out using an energy and force convergence criteria of $1 \mathrm{e}^{-25}$. Lastly, the $\mathrm{TJ}$ structure was relaxed again at $300 \mathrm{~K}$ using the canonical ensemble 
(NVT) for $1 \mathrm{~ns}$. Afterwards, the intrinsic properties relevant to stability for the TJs were examined and will be discussed in detail later.

Table 1. Details of grain boundary triple junctions along with the CSL and the misorientation angle $(\theta)$ for each GB interface.

\begin{tabular}{cccc}
\hline Triple Junction & GB \#1 $(\Sigma, \boldsymbol{\theta})$ & $\mathbf{G B} \# \mathbf{2}(\Sigma, \boldsymbol{\theta})$ & $\mathbf{G B} \# \mathbf{3}(\Sigma, \boldsymbol{\theta})$ \\
\hline$\Sigma 3-\Sigma 3-\Sigma 9$ & $\Sigma 9(114), 38.94^{\circ}$ & $\Sigma 3(112), 70.53^{\circ}$ & $\Sigma 3(112), 70.53^{\circ}$ \\
$\Sigma 3-\Sigma 9-\Sigma 27$ & $\Sigma 27(552), 148.41^{\circ}$ & $\Sigma 9(221), 141.06^{\circ}$ & $\Sigma 3(112), 70.53^{\circ}$ \\
$\Sigma 3-\Sigma 11-\Sigma 33$ & $\Sigma 33(225), 58.99^{\circ}$ & $\Sigma 11(113), 50.48^{\circ}$ & $\Sigma 3(112), 70.53^{\circ}$ \\
$\Sigma 3-\Sigma 19-\Sigma 57$ & $\Sigma 57(558), 82.95^{\circ}$ & $\Sigma 19(116), 26.52^{\circ}$ & $\Sigma 3(112), 70.53^{\circ}$ \\
$\Sigma 3-\Sigma 27-\Sigma 81$ & $\Sigma 81(447), 77.88^{\circ}$ & $\Sigma 27(115), 31.59^{\circ}$ & $\Sigma 3(112), 70.53^{\circ}$ \\
$\Sigma 3-\Sigma 33-\Sigma 99$ & $\Sigma 99(7710), 89.42^{\circ}$ & $\Sigma 33(118), 20.05^{\circ}$ & $\Sigma 3(112), 70.53^{\circ}$ \\
\hline
\end{tabular}

The role of the atomic structure surrounding the $\mathrm{TJ}$ on the binding energetics of point defects (vacancy and self-interstitial atom) and various solute atoms ( $\mathrm{H}, \mathrm{He}, \mathrm{P}$ and $\mathrm{V}$ ) were assessed by calculating the formation/binding energies. The solute atoms studied in this work represent both the interstitial $(\mathrm{H}$ and $\mathrm{He}$ ) and substitutional ( $\mathrm{P}$ and $\mathrm{V}$ ) impurities for $\alpha-\mathrm{Fe}$. The substitutional solute atom ( $\mathrm{P}$ and $\mathrm{V}$ ) was introduced by replacing an Fe atom at the atomic site $\alpha$ (Figure 1b). The substitutional defect (vacancy, $\mathrm{P}$ and V) formation energy at the site $\alpha$ can be expressed in the following manner:

$$
E_{f}^{\alpha}=E_{t}^{\alpha}-E_{t}+E_{c o h}^{F e}-E_{c o h}^{\alpha}
$$

Here, $E_{t}^{\alpha}$ and $E_{t}$ are the total energies of the TJ simulation cell with and without the substitutional defect, respectively. The $E_{c o h}^{F e}$ and $E_{c o h}^{\alpha}$ terms are the cohesive energies of the Fe and the solute atom (in the case of a vacancy, $E_{c o h}^{\alpha}=0$ ), respectively. It is useful to compare the substitutional defect formation energy in the vicinity of the TJ configuration $\left(E_{f, T J}^{\alpha}\right)$ to that in the $\operatorname{bulk}\left(E_{f, b u l k}^{\alpha}\right)$.

$$
E_{b}^{\alpha}=E_{f, b u l k}^{\alpha}-E_{f, T J}^{\alpha}
$$

The $E_{f, b u l k}^{\alpha}$ for vacancy, $\mathrm{V}$ and $\mathrm{P}$ were $1.75,-0.58$ and $2.05 \mathrm{eV}$, respectively, see [65,71]. In the case of an interstitial solute atom ( $\mathrm{H}$ and $\mathrm{He}$ ), a slightly different methodology was employed. Since a single instance may not obtain the minimum formation/binding energy, thirty six different starting positions were randomly selected within a sphere of radius $\sqrt{3} / 2 a_{0}\left(a_{0}\right.$ is the lattice parameter) around the atomic site $\beta$. The formation and binding energies for an interstitial 
defect (SIA, $\mathrm{H}$ and $\mathrm{He}$ ) at the atomic site $\beta$ in the vicinity of the TJ (Figure 1c) can be expressed in the following manner:

$$
\begin{aligned}
& E_{f}^{\beta}=E_{t}^{\beta}-E_{t}-E_{c o h}^{\beta} \\
& E_{b}^{\beta}=\frac{1}{36} \sum_{i}\left(E_{f, \text { bulk }}^{\beta}-E_{f, T J}^{\beta}\right)_{i}
\end{aligned}
$$

where, $E_{c o h}^{\beta}$ is the cohesive energy of the solute atom and $E_{t}^{\beta}$ is the total energy of the TJ simulation cell in the presence of an interstitial atom. A similar methodology was employed by Tschopp et al. [72] to obtain the statistical information regarding the binding energetics of a $\mathrm{He}$ cluster around Fe GBs. In order to study the effect of the TJ atomic arrangement on the vacancy migration behavior, the NEB method [43] was employed. The variation in the diffusion of the vacancy was examined for all the atomic sites within a radius of $5 \AA$ of the TJ (i.e., approximately $25 \mathrm{NEB}$ simulations for each TJ). This was carried out by the creation of two distinct $\mathrm{TJ}$ structures that serve as the initial and final atomic positions for the vacancy migration along the TJ line. The quick-min optimization scheme (refer [73] for more details) with a force tolerance criteria of 1e-3 and 12 intermediate images were employed to find the minimum energy pathway (MEP) for the migration of a vacancy along the TJ line.

\section{Triple junction atomic structure and thermodynamic properties}

Understanding the role of TJs on the structural stability and solute binding behavior is critical in engineering $\mathrm{NC}$ materials resistant to grain growth and radiation induced segregation. Hence, we focus on characterizing the $\mathrm{TJ}$ atomic structures by measuring the hydrostatic stress field and the variation in the potential energy due to the formation of TJs. These structural properties provide a basis for defining other intrinsic properties, such as the TJ strain energy evolution and the resolved line tension that are critical in understanding the structural stability of the TJ. For instance, the hydrostatic stress field is further used to compute the resolved line tension acting at the $\mathrm{TJ}$, and the TJ strain energy evolution provides a measure of the energy localization around the TJ. Note that we have also computed other thermodynamic variables such as the excess energy and volumetric strain due to the formation of the TJ, see Appendix A for further details.

The atomic distribution of the hydrostatic stress fields for the various TJs provides an assessment of the stress buildup and outlines the dislocation structure of the GBs intersecting at the TJ (Figure 2). The hydrostatic stress accumulated near the $\Sigma 3-\Sigma 3-\Sigma 9$ TJ (Figure 2a) was 
higher when compared to the stress accumulated near the $\Sigma 3-\Sigma 9-\Sigma 27 \mathrm{TJ}$ (Figure $2 \mathrm{~b}$ ). This clearly highlights the importance of the local atomic arrangement surrounding the TJ compared to the constituent GBs. For instance, the magnification of hydrostatic stress near the $\Sigma 3-\Sigma 3-\Sigma 9 \mathrm{TJ}$ (Figure 2a) far exceeded the maximum hydrostatic stress observed along the GBs. On the other hand, in the case of the $\Sigma 3-\Sigma 9-\Sigma 27 \mathrm{TJ}$ (Figure 2b), there was no change in the hydrostatic stress distribution near the TJ when compared to the behavior observed along the constituent GBs. Similarly, the stress accumulation far exceeded the stress observed along the constituent GBs in the $\Sigma 3-\Sigma 11-\Sigma 33 \mathrm{TJ}$ (Figure 2c). A plausible explanation for this behavior can be the intersection of high energy interfaces ( $\Sigma 11$ and $\Sigma 33 \mathrm{GBs}$ ) that causes a significant change in the nature of the TJ behavior resulting in a GB dominated behavior. On the other hand, the stress buildup for the $\Sigma 3-\Sigma 27-\Sigma 81 \mathrm{TJ}$ and other TJs (see Figures $2 \mathrm{~d}$, e and f) was found to be far lower when compared to the constituent GBs. The variation in atomic hydrostatic stress near the TJ was further utilized to characterize the resolved line tension. The approximate resolved line tension $\left(\gamma_{r}\right)$ acting at the TJ was calculated as:

$$
\gamma_{r}=\sum_{i=1}^{3} \gamma_{i} l_{x}^{i}
$$

where, $l_{x}^{i}$ is the length of the unit vector tangential to the GB plane and the surface tension for the intersecting GBs $\left(\gamma_{i}\right)$ was estimated by averaging the normal and tangential stresses acting over a region of $\pm 20 \AA$ normal to the interfaces. The line tension at the TJ acts as a driving force for the grain growth [74]. In other words, a higher resolved line tension at the TJ results in greater mobility of the $\mathrm{NC}$ material, as the overall microstructural mobility is greatly weighed by the TJ behavior. Therefore, quantifying the resolved line tension across various TJs is vital in the attempts to engineer $\mathrm{NC}$ material with improved structural stability. Figure 3a shows the resolved line tension trend for a majority of the TJs, which was found to vary within 0.5 to 1 $\mathrm{N} / \mathrm{m}$, except for the $\Sigma 3-\Sigma 33-\Sigma 99 \mathrm{TJ}(2.4 \mathrm{~N} / \mathrm{m})$. In the case of the $\Sigma 3-\Sigma 33-\Sigma 99 \mathrm{TJ}$, the significantly higher resolved line tension can be attributed to the intersection of high energy interfaces at the TJ. From a microstructure stability point of view, the physical ramifications of a higher line tension suggests a higher TJ mobility, i.e., TJs with a high line tension will result in a loss of structural stability [74]. 


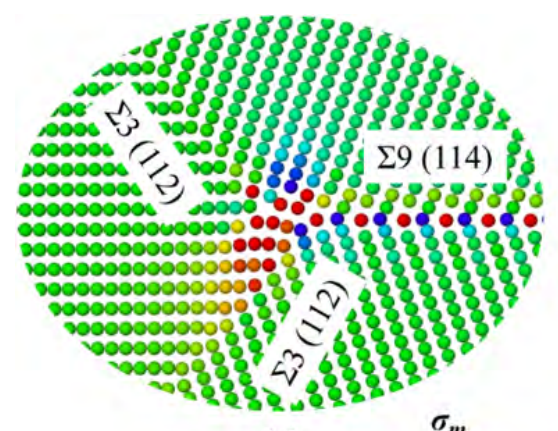

(a)

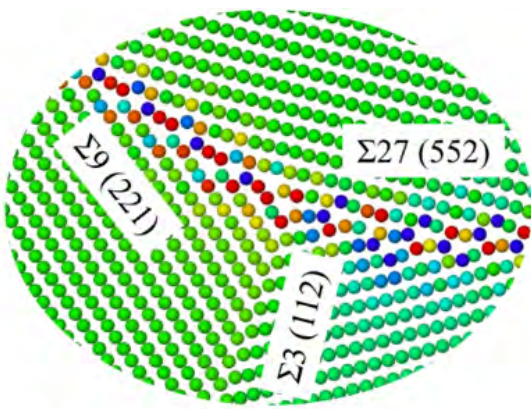

(b)

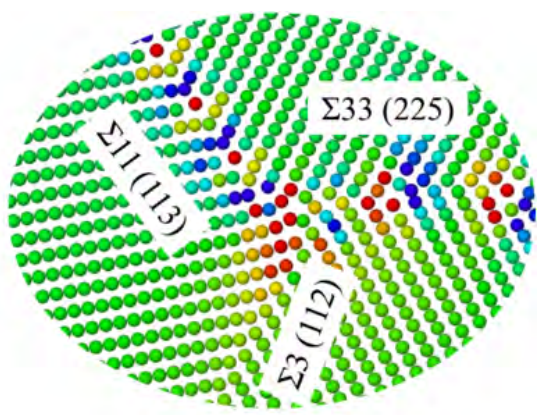

(c)

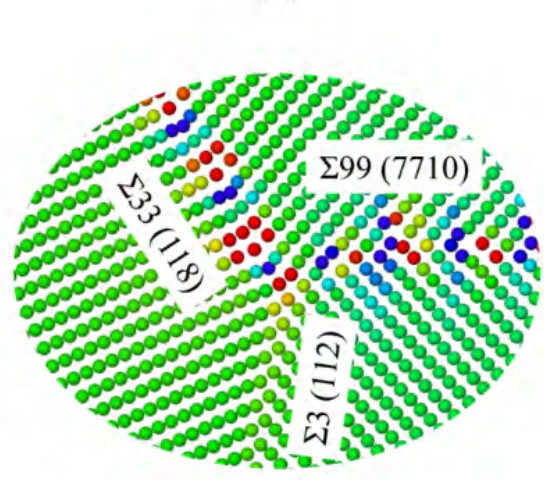

(f)

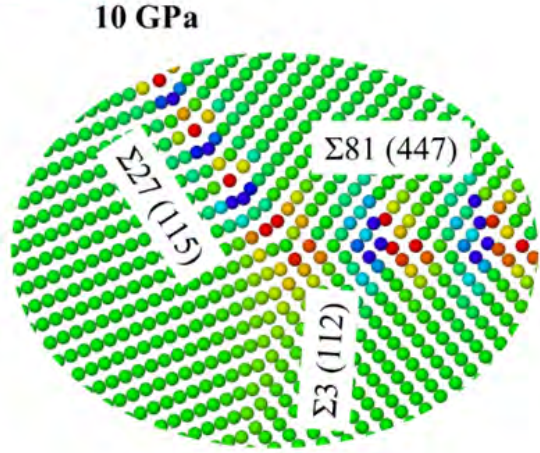

(e)

Figure 2: The minimized atomic configuration of: a) $\Sigma 3-\Sigma 3-\Sigma 9$, b) $\Sigma 3-\Sigma 9-\Sigma 27$, c) $\Sigma 3-\Sigma 11-\Sigma 33$, d) $\Sigma 3-\Sigma 19-\Sigma 57$, e) $\Sigma 3-\Sigma 27-\Sigma 81$ and f) $\Sigma 3-\Sigma 33-\Sigma 99$ TJs. Atoms in the perfect BCC lattice are depicted as green, while the defected atoms along the GB and the TJ are colored according to the variation in hydrostatic stress.

Next, the strain energy evolution at the TJ as a function of radial distance $\left(E_{T J}(r)\right)$ was calculated using the following expression:

$$
E_{T J}(r)=E_{0} \ln \left(\frac{r}{b}\right)+E_{\text {core }}
$$

where, $E_{\text {core }}$ is the TJ core energy, $b$ is the magnitude of the Burger's vector $\left(\vec{b}=\frac{a_{0}}{2}[111]\right), E_{0}$ is a constant that depends on the elastic properties of the material, $r$ is the radius of the cylinder and $E_{T J}$ is the sum of excess energies (calculated by subtracting the cohesive energy contribution) of all the atoms that lie within a radius of $r$ from the TJ. This metric can be employed to assess the strain energy localization around the TJ, similar to that of an individual dislocation core [75]. Due the elastic anisotropy [76,77] and the breakdown of continuum elasticity near the defect core, the core energy term was introduced in Equation 4 (refer to [75]). 
Figure $3 \mathrm{~b}$ shows the TJ strain energy evolution as a function of the radial distance for a few selected TJs. As shown in Figure 3b, the $\Sigma 3-\Sigma 3-\Sigma 9$ and $\Sigma 3-\Sigma 11-\Sigma 33$ TJs have the highest strain energy localization around the Burger's vector length $(r \approx b)$, i.e., at the TJ core (also refer to Table 2). Further, the approximate slope $(m)$ of Figure $3 b$ computed for $r>b$ indicates the degree of constituent GB contributions towards the total strain energy evolution. In other words, a high $m$ value corresponds to a significantly high contribution of the strain energy from the constituent GBs as compared to the TJ. For instance, in the case of the $\Sigma 3-\Sigma 3-\Sigma 9 \mathrm{TJ}$, the rate of strain energy accumulation (slope of the curves $(\mathrm{m})$ in Figure $3 \mathrm{~b}$ ) reduces drastically with increasing distance from the TJ $(r>b)$, suggesting a reduced GB contribution. On the contrary, the rate of strain energy accumulation for the $\Sigma 3-\Sigma 11-\Sigma 33 \mathrm{TJ}$ remained high consistently with increasing distance from the TJ, therefore a strong GB contribution can be inferred. In summary, the strain energy evolution helps in understanding the following issues: a) the strain energy distribution due to the formation of a TJ (i.e., due to the localized TJ structure) and b) the competition between TJ and GB dominated energetics.

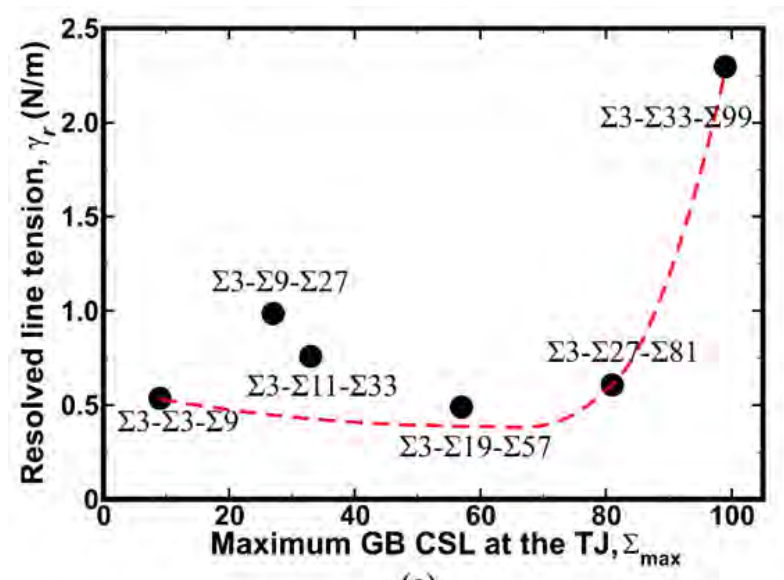

(a)

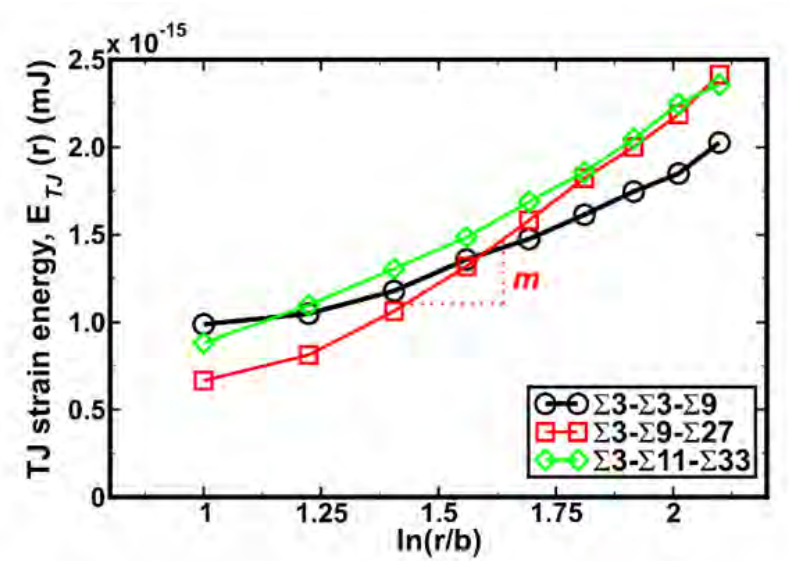

(b)

Figure 3: (a) The resolved line tension at the TJ and (b) the evolution of the TJ strain energy as a function of the logarithmic distance from the TJ for a few selected TJs. 
Table 2. The TJ core energy, $\boldsymbol{E}_{\text {core }}$ and the average slope, $\boldsymbol{m}$ obtained from the TJ strain energy evolution curve as described in Figure $3 b$ for the various TJs.

\begin{tabular}{ccc}
\hline Triple Junction & $\begin{array}{c}\text { TJ core energy, } \\
\boldsymbol{E}_{\text {core }} \boldsymbol{(}\left(\times \mathbf{1 0}^{-\mathbf{1 5}} \mathbf{m J}\right)\end{array}$ & $\begin{array}{c}\text { Slope of the TJ strain energy } \\
\text { versus } \mathbf{l n}(\mathbf{r} / \mathbf{b}), \\
\boldsymbol{m},\left(\times \mathbf{1 0}^{-15} \mathbf{m J}\right)\end{array}$ \\
\hline$\Sigma 3-\Sigma 3-\Sigma 9$ & 0.99 & 0.52 \\
$\Sigma 3-\Sigma 9-\Sigma 27$ & 0.67 & 1.16 \\
$\Sigma 3-\Sigma 11-\Sigma 33$ & 0.89 & 0.85 \\
$\Sigma 3-\Sigma 19-\Sigma 57$ & 0.60 & 0.46 \\
$\Sigma 3-\Sigma 27-\Sigma 81$ & 0.49 & 1.00 \\
$\Sigma 3-\Sigma 33-\Sigma 99$ & 0.61 & 1.07 \\
\hline
\end{tabular}

\section{Correlating the triple junction thermodynamic properties to point defects binding energetics}

Radiation damage creates lattice point defects, which can alter the physical and mechanical properties of the material through the nucleation of defects such as defect clusters, defectimpurity complexes, voids and defect-solute clusters. Thus, quantifying how point defects, such as vacancy and SIA, interact with defect sinks (TJ and the constituent GBs) is critical. For instance, the stability of a nuclear structural material relies on its effectiveness in absorbing point defects through various microstructural sinks (GBs and TJs). Further, vacancies and SIA's play an important role in the solute kinetics behavior in the vicinity of the TJs and their constituent GBs. Therefore, before turning our attention to solute atom $(\mathrm{H}, \mathrm{He}, \mathrm{V}$ and $\mathrm{P})$ energetics per se, we first focus on the vacancy and SIA binding behavior near the TJ.

The spatial variation in the vacancy binding energy $\left(E_{b}^{v a c}\right)$ near TJs is shown in Figure 4. For the sake of brevity, the vacancy binding energy of $\Sigma 3-\Sigma 3-\Sigma 9$ and $\Sigma 3-\Sigma 33-\Sigma 99$ TJs are presented herein. The effect of TJs on the binding tendency for atomic sites within the grain interior was found to be negligible, i.e., the atoms further away from the TJs and constituent GBs are colored white ( $0 \mathrm{eV}$ vacancy binding energy), indicating that there is no difference in formation energy compared to the pristine BCC lattice (see Figure 4). Further, the atomic sites with higher vacancy binding energy were found to be clustered near regions of high atomic hydrostatic stresses (Figures 2 and 4). Also, as shown in Figure 4, there is a clear difference in the numbers of preferred binding sites $\left(E_{b}^{v a c}>0\right)$ in the vicinity of the TJ between the $\Sigma 3-\Sigma 3-\Sigma 9$ and the $\Sigma 3$ $\Sigma 33-\Sigma 99$ TJs. The $\Sigma 3-\Sigma 3-\Sigma 9$ TJ has preferred vacancy sites concentrated in a very small region around the triple point within a $3 \AA$ radius, indicating the extent of the TJ elastic strain field. On 
the other hand, the $\Sigma 3-\Sigma 33-\Sigma 99 \mathrm{TJ}$ has more stable vacancy sites $\left(E_{b}^{v a c}>0\right)$ away from the TJ along the constituent GBs (Figure 4b).

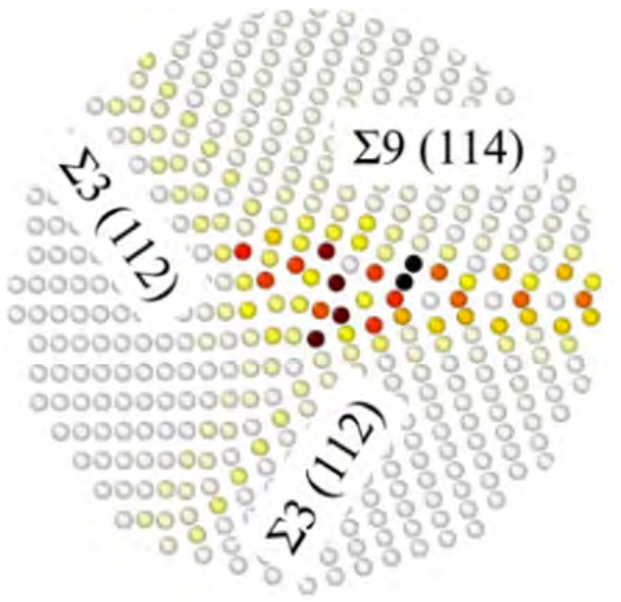

(a)

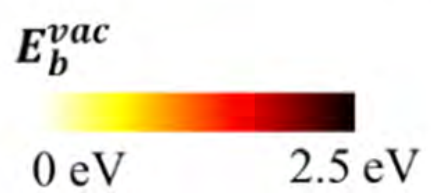

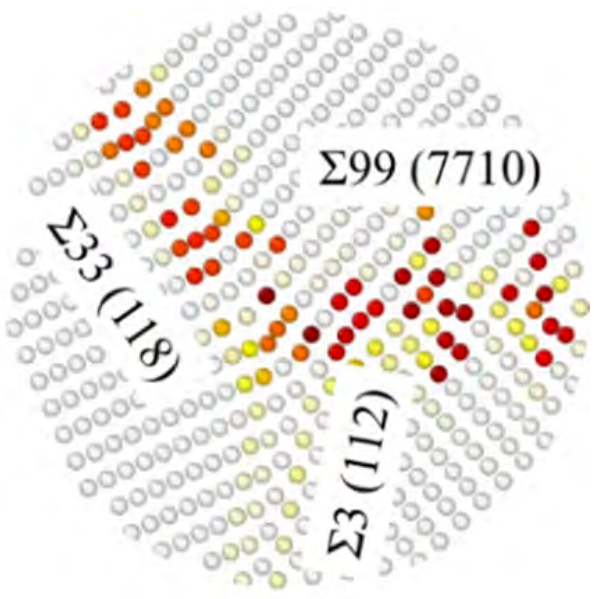

(b)

Figure 4: The atomic variation in vacancy binding energy near a) $\Sigma 3-\Sigma 3-\Sigma 9$ and b) $\Sigma 3-\Sigma 33-\Sigma 99$ TJs. The atoms were colored based on the vacancy binding energy at each site. White atoms correspond to the bulk binding energy $(\sim 0 \mathrm{eV})$ and black atoms represent the minimum vacancy binding energy of $2.5 \mathrm{eV}$.

To quantify the spatial extent of the vacancy and SIA binding energies relative to the bulk, we focus our attention on the mean vacancy $\left(\bar{E}_{b}^{v a c}\right)$ and SIA $\left(\bar{E}_{b}^{S I A}\right)$ binding energies as a function of radial distance from the TJ, i.e., the mean binding energy for all the atomic sites that lie within small concentric regions ( $2 \AA$ intervals) from the TJ. A total of 10 concentric bins were defined $\left(r_{i-1} \leq r \leq r_{i}\right)$ up to a distance of $20 \AA$ from the TJ. Figure 5 plots the mean vacancy $\left(\bar{E}_{b}^{v a c}\right)$ and SIA $\left(\bar{E}_{b}^{S I A}\right)$ binding energies as a function of radial distance from the TJ. Here, the computed mean binding energies are used to comprehend the interplay between the GB and TJ on the binding strength of point defects. For example, the vacancy and SIA mean binding energies for a majority of the TJs converged to the bulk formation energy at a length of approximately $15 \AA$ from the TJ (where, $E_{f}^{\text {bulk }}=1.75 \mathrm{eV}$ ), i.e., the length scale over which there is a significant influence of the TJ on the point defect binding energetics. Further, there appears to be a correlation between the variation in the mean binding energies (vacancy and SIA) as a 
function of radial distance from the TJ and the evolution of the TJ strain energy, compare Figures 5 and $3 b$. For example, the $\Sigma 3-\Sigma 3-\Sigma 9$ TJ had a highly localized TJ core energy (Figure $3 b)$ and, hence, a high preference to accommodate a vacancy near the TJ core (within $5 \AA$ radially). On the other hand, the $\Sigma 3-\Sigma 33-\Sigma 99 \mathrm{TJ}$ has lower concentration of energetically favorable sites near the TJ, which is in part due to an increasing influence of the constituent GBs on the TJ strain energy (Figure 3b). Further, similar behavior was observed in the case of SIA (Figure $5 b$ ). However, there was a higher preference for accommodating SIA in contrast to vacancies around the TJs, which is in agreement with previous simulations, e.g. Ref [78]. Subsequently, there is a corresponding larger energetic driving force for SIA to bind around the $\mathrm{TJ}$ in $\alpha$-Fe over vacancies. Overall, the binding energetics of point defects highlights the critical interplay between the GB and TJ dominated behavior.

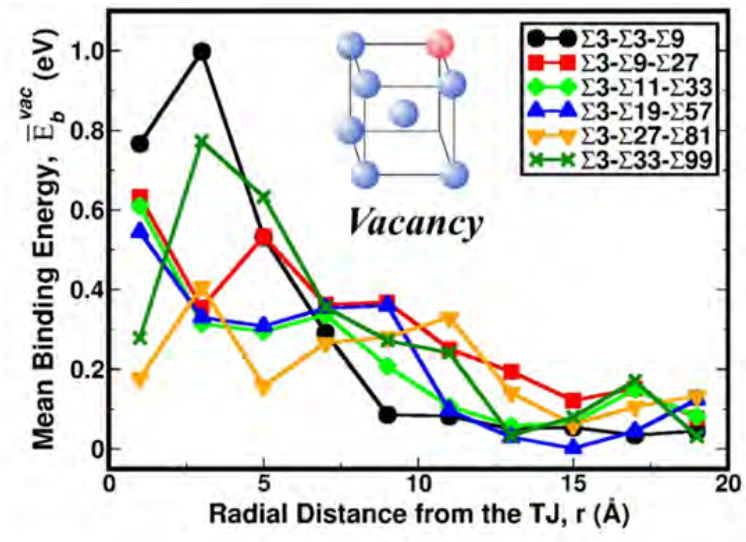

(a)

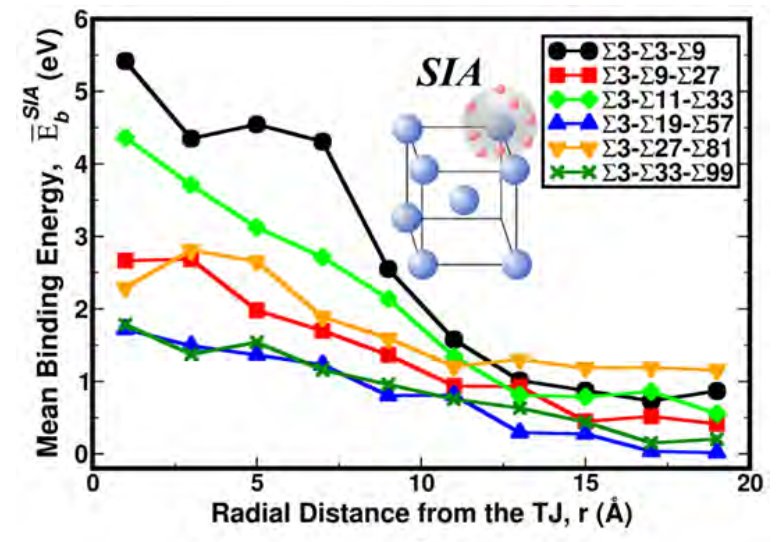

(b)

Figure 5: The mean (a) vacancy and (b) SIA binding energies as a function of radial distance from the TJ. A total of 10 concentric bins were defined $\left(r_{i-1} \leq r \leq r_{i}\right)$ up to $20 \AA$ from the TJ.

\section{Vacancy migration behavior along the triple junction}

During radiation, the impact of high energy particles on the structural material induces a large number of defects and the GBs and TJs act as sinks for these defects. The increased volume fraction of grain boundaries in a NC material allows for the effective absorption of defects at the interface, lowering the probability of aggregation of defects, thereby, improving the resistance to radiation damage over coarse grained materials $[2,8,79,80]$. However, the increased volume fraction of the TJs in NC material can significantly alter the material behavior [42,81-83]. Further, as mentioned earlier, the microstructural stability is critical for broader applicability of NC materials. Therefore, it is critical to evaluate the role of TJs and their constituent GBs on the 
structural stability and material behavior. The effect of the interplay between a GB and the TJ on the grain growth has been discussed in previous work $[84,85]$. For example, it has been shown that below a certain critical grain size, the immobile TJs can create a drag effect to the microstructural growth. Here, we characterize the mass transport properties of the TJs by quantifying the activation energy for self-diffusion, i.e., the sum of vacancy formation and migration energies for various TJs:

$$
Q_{T J}=E_{f, T J}^{v a c}+E_{m i g, T J}^{v a c}
$$

where, $E_{f, T J}^{v a c}$ and $E_{m i g, T J}^{v a c}$ are the vacancy formation and migration energies in the vicinity of the TJ, respectively. A large degree of site to site variation was observed in the activation energy for vacancy migration along the TJ. Thus, the probability density function (PDF or probability density of events) was quantified to understand the likelihood of observing various vacancy activation energy (Figures 6a-f) for the TJs examined here. Examining the PDF of activation energies reveals that some of these differences are a clear consequence of the atomic structure of the TJ, see Figures 6a-f. To further statistically quantify the scatter in the activation energy, we focused on the full width half maximum (FWHM), i.e. the spread in the activation energy across which the probability density falls to half its peak value (Figures 6a-f). This indicates statistically relevant vacancies of activation energies for the various TJ, as the probability of occurrence is high $(\sim 68 \%)$. Furthermore, in this work the trend between the mean activation energy for diffusion and the resolved line tension (Figure 6g) was examined to provide a measure of the mass transport behavior for a NC material. Figure $6 \mathrm{~g}$ clearly highlights the inverse dependence of the mean activation energy for vacancy diffusion on the resolved line tension at the TJ (Figure $6 \mathrm{~g}$ ). In other words, the TJ with a higher line tension will thermodynamically be more unstable. 

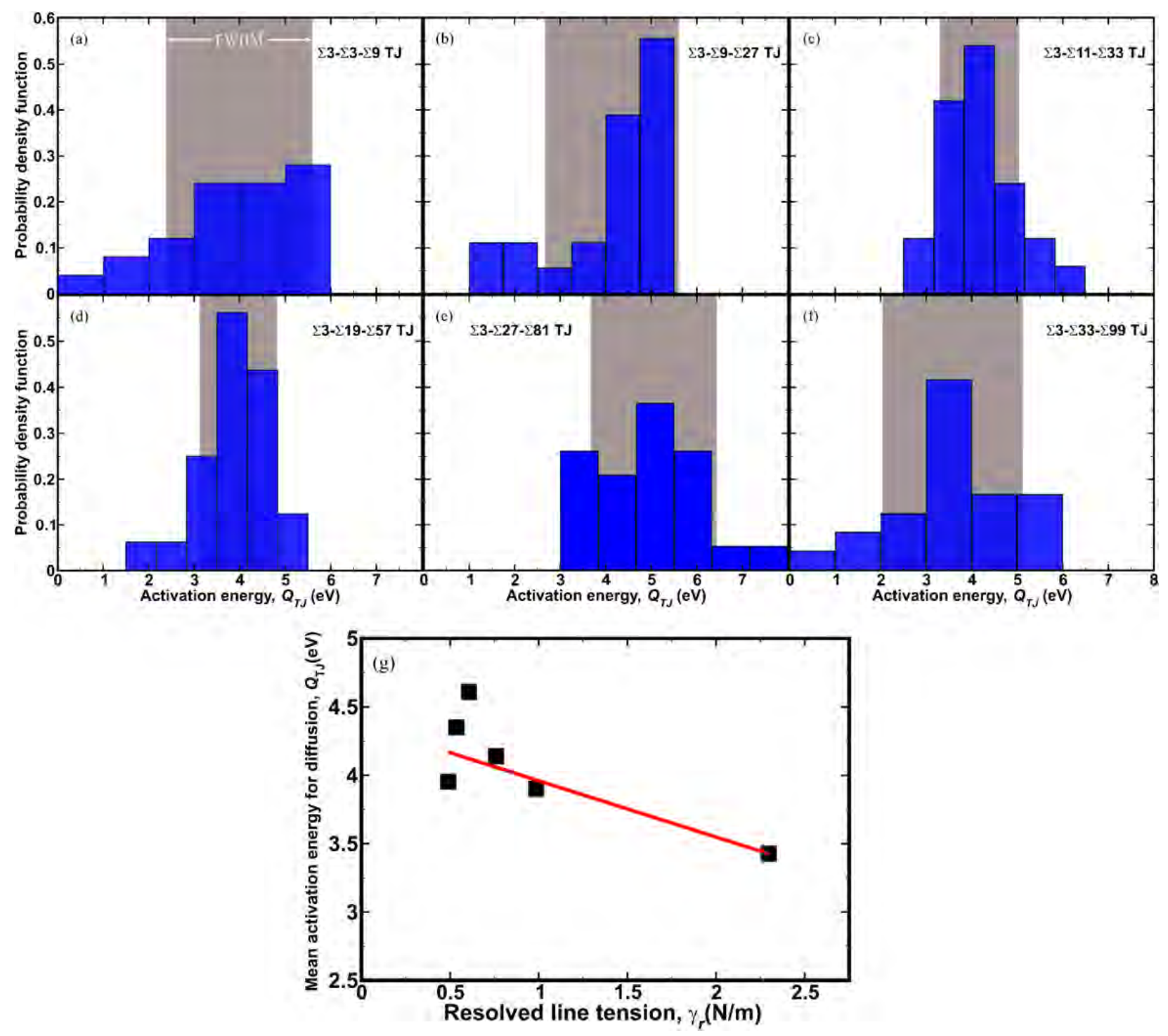

Figure 6: a-f) The vacancy diffusion activation energy probability density function for various TJs; and g) the variation in the mean activation energy for diffusion against the resolved line tension quantified earlier for the TJs.

\section{Solute (H, He, V and $\mathrm{P})$ binding behavior}

In order to engineer next generation radiation tolerant material, it is imperative to understand the energetics associated with segregation of solutes $(\mathrm{H}, \mathrm{He}, \mathrm{V}$ and $\mathrm{P})$ around defects, such as GBs and TJs. Therefore, probing the binding behavior for a variety of TJs can provide a fundamental understanding of the relationship between the binding energetics and the atomic structure. Hence, we first studied the spatial variation in binding energies at two TJs as a function of different impurities. This helps in qualitatively assessing the propensity of the TJ and their 
constituent GBs to the various impurity atoms both at the substitutional and interstitial configurations. The $\Sigma 3-\Sigma 3-\Sigma 9$ and $\Sigma 3-\Sigma 11-\Sigma 33$ TJs were chosen to highlight the difference between the binding energetics for the various substitutional and interstitial impurity atoms. As discussed earlier, for an interstitial element, such as a $\mathrm{H}$ or $\mathrm{He}$ atom, a single instance is not sufficient to understand the formation/binding energy behavior. Therefore, thirty-six different starting positions were randomly selected within a sphere of radius $\sqrt{3} / 2 a_{0}\left(a_{0}\right.$ is the lattice parameter) around each atomic site in the vicinity of the TJ (Figure 1c).

Figure 7 illustrates the site to site mapping of the binding energy for the $\Sigma 3-\Sigma 3-\Sigma 9$ and $\Sigma 3$ $\Sigma 11-\Sigma 33$ TJs for the various solute atoms $(\mathrm{H}, \mathrm{He}, \mathrm{P}$ and $\mathrm{V})$. For all the impurity atoms, the binding energy was found to rapidly diminish towards $0 \mathrm{eV}$ as we moved away from the $\mathrm{TJ}$ and into the bulk lattice. A few key observations can be drawn from Figure 7: (1) in the case of both $\mathrm{H}$ and $\mathrm{He}$ atoms, a highly localized region $(<5 \AA)$ of extremely stable binding sites were found in the vicinity of the TJs, indicating that $\mathrm{H}$ and He prefer to bind around the TJs compared to the constituent GBs; (2) in the case of an interstitial $\mathrm{H}$ and $\mathrm{He}$ atom, the most energetically favorable binding sites were found to be clustered near regions of high hydrostatic stresses, compare Figures 2 and 7; (3) the order of binding behavior at the $\Sigma 3-\Sigma 3-\Sigma 9$ and $\Sigma 3-\Sigma 11-\Sigma 33$ TJs listed from highest to lowest is Fe-He, Fe-P, Fe-V and Fe-H, respectively; (4) in the case of $\mathrm{P}$ which is known to cause temper embrittlement in steel [86], the TJs show a strong affinity for P to bind near the TJ core with a small fraction of binding sites away from the TJ core and (5) in the case of the substitutional $\mathrm{V}$ atom, only a small number of sites around the TJs were energetically favorable for the impurity atom to bind. Interestingly, along the constituent GBs, the V atom had negligible energetic preference (in agreement with previous simulations, e.g. Ref [60]). Overall, a low number of energetically favorable binding sites near the TJs were observed for both the $\mathrm{P}$ and $\mathrm{V}$ atoms compared to other impurities studied here. A plausible explanation could be that the large atomic size of these substitutional atoms limits the number of preferable binding sites around defects. 


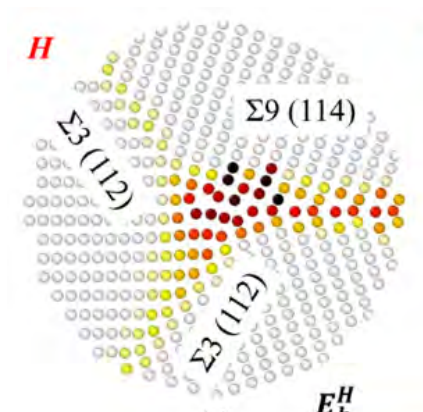

(a)

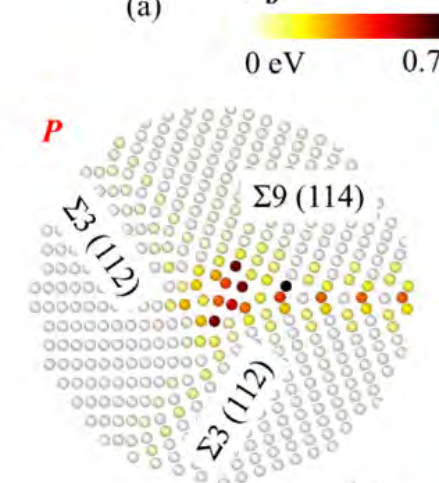

(e)

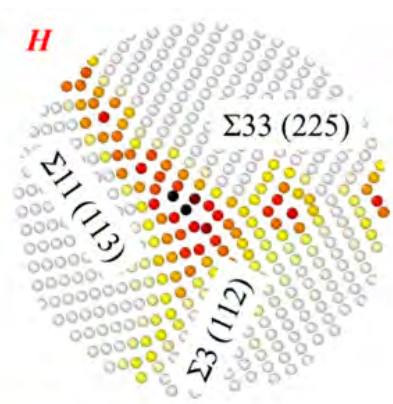

(b)

$0.75 \mathrm{eV}$

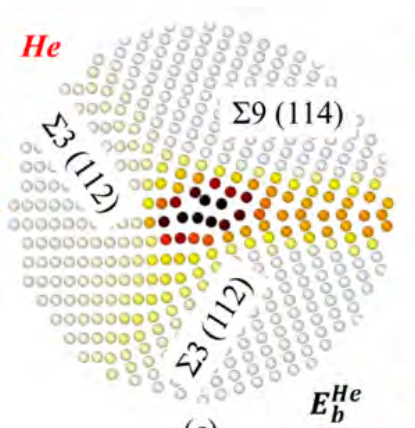

(c)

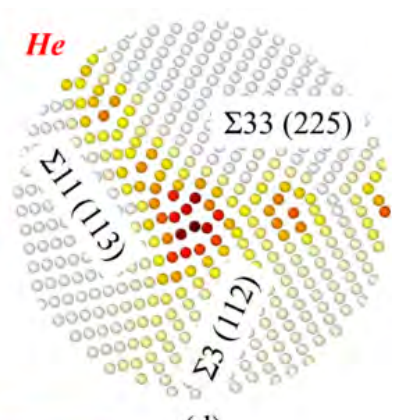

(d)

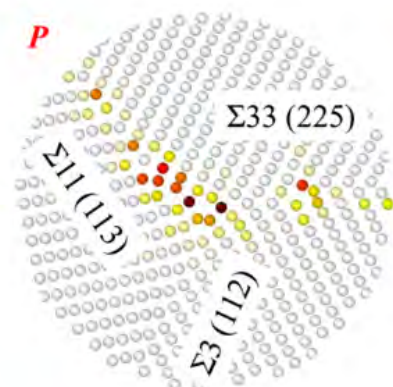

(f)

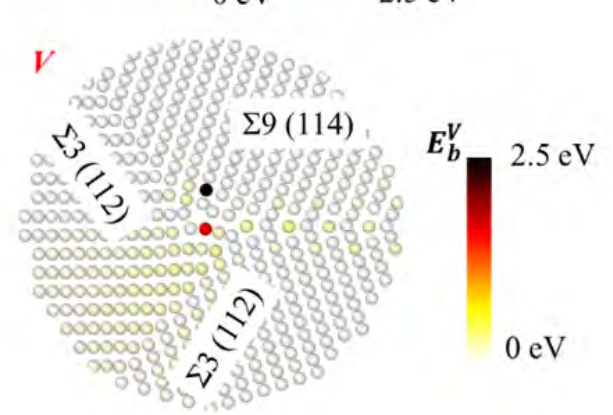

(g)

$0 \mathrm{eV} \quad 2.5 \mathrm{eV}$

Figure 7: The atomic variation in solute binding behavior was examined for the $\Sigma 3-\Sigma 3-\Sigma 9$ and $\Sigma 3-\Sigma 11-\Sigma 33$ TJs for (a-b) hydrogen, (c-d) helium, (e-f) phosphorous and (g) the $\Sigma 3-\Sigma 3-\Sigma 9$ TJ for vanadium. The white atoms correspond to the bulk binding energy $(\sim 0 \mathrm{eV})$ and the black atoms represent the maximum solute binding energy. In the case of hydrogen, it was $0.75 \mathrm{eV}$ and 2.5 $\mathrm{eV}$ for the remaining solute examined here. Positive binding energies imply a favorable binding behavior, thus the sites of maximum binding energy would act as the sink sites for solute atoms.

Next, we investigate the binding behavior of various impurities around the TJ and their constituent GBs by computing the mean binding energy for all the sites that lie within a small radial region, refer to Figure 8. These binding energies as shown in Figure 8 suggest that (1) The $\Sigma 3-\Sigma 3-\Sigma 9$ TJ was found to possess the highest binding strength for all the interstitial and substitutional impurity atoms among the TJs examined here; (2) similarly, the $\Sigma 3-\Sigma 9-\Sigma 27 \mathrm{TJ}$ exhibits the least binding characteristics as compared to the various TJs examined herein; (3) a strong correlation between the solute binding behavior and the evolution of the TJ core energy was found (refer to Figures $3 \mathrm{~b}$ and 8); (4) in the case of the $\Sigma 3-\Sigma 3-\Sigma 9$ TJ, the mean binding strength for all the solute atoms examined here was found to decay rapidly with increasing 
distance from the TJ core, which is in agreement with the trend for the TJ strain energy (Figure $3 b)$; (5) in general, the mean binding strength for all the solute atoms across the various TJs was found to converge towards $0 \mathrm{eV}$ around a distance of $10-15 \AA$ and (6) for the $\Sigma 3-\Sigma 27-\Sigma 81 \mathrm{TJ}$, the mean binding energy remained high $(>0 \mathrm{eV})$ with an increasing distance from the TJ, due to the availability of stable binding sites along the constituent GBs.

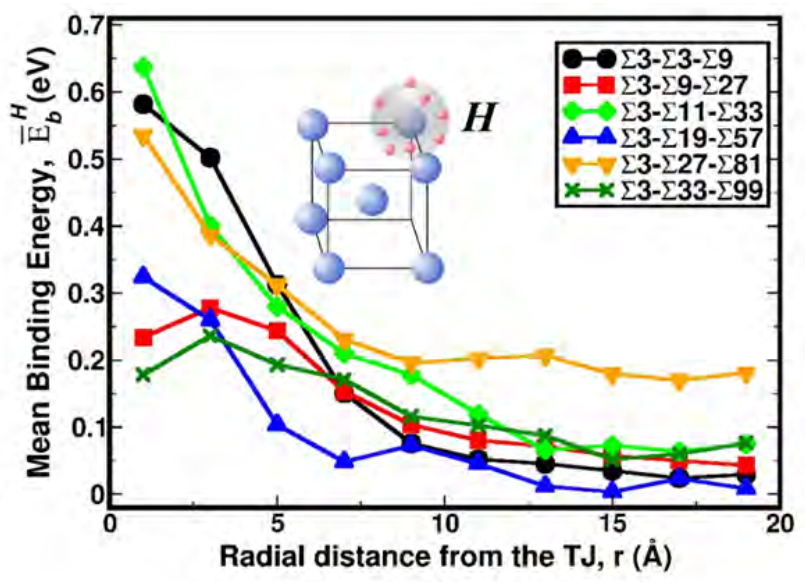

(a)

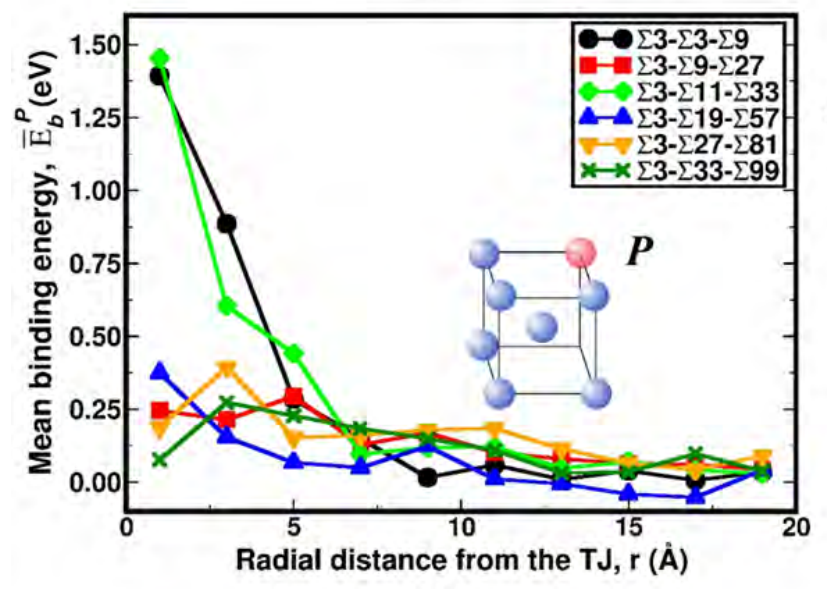

(c)

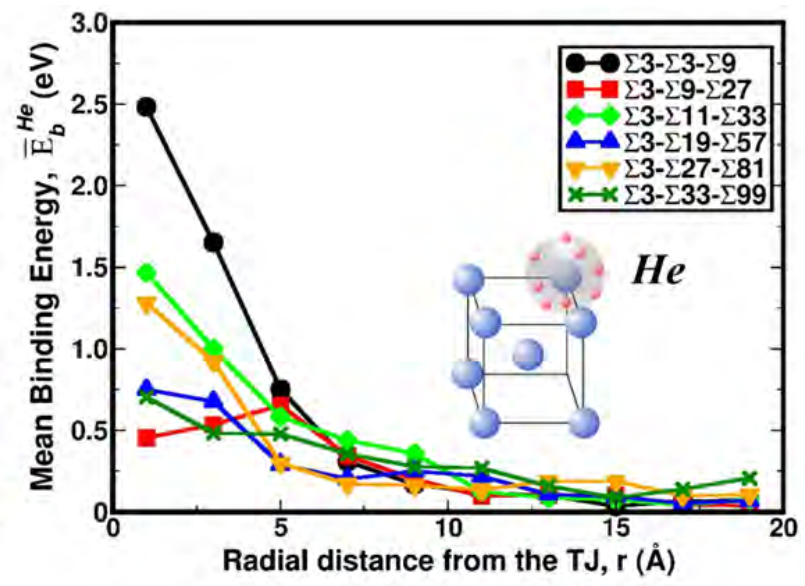

(b)

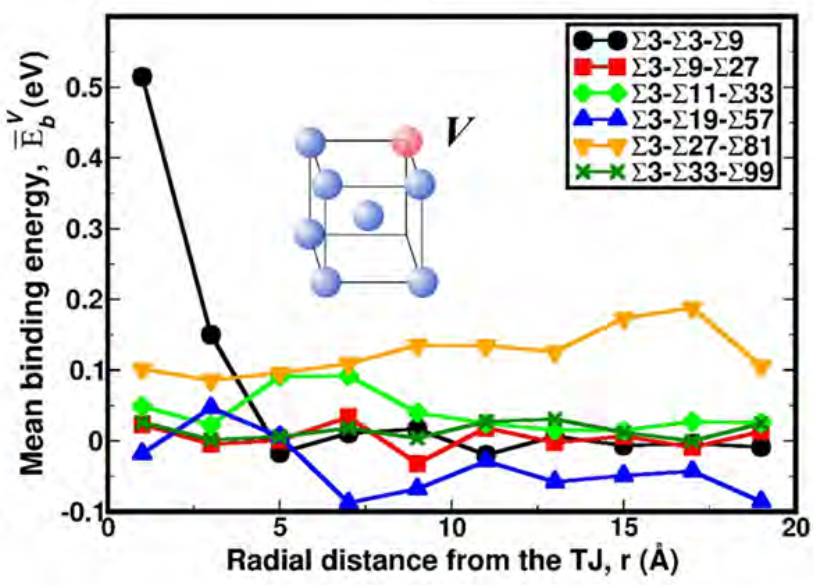

(d)

Figure 8: The mean binding energy as a function of radial distance from the $\mathrm{TJ}$ for the following solute atoms: a) Hydrogen; b) Helium; c) Phosphorous and d) Vanadium. A total of 10 concentric bins were defined $\left(r_{i-1} \leq r \leq r_{i}\right)$ up to $20 \AA$ from the TJ.

\section{Implications of solute binding behavior around the TJ region}

The length scale over which the TJ influences the solute binding behavior was examined for the $\Sigma 3-\Sigma 3-\Sigma 9$ and $\Sigma 3-\Sigma 11-\Sigma 33$ TJs (Figure 9). This was determined by measuring the distance 
from the TJ along the constituent GBs and the three $\{110\}$ planes intersecting the $\mathrm{TJ}$ core where a distinct change in the solute binding behavior was observed. This is because the atomic sites that lie within the envelope of the TJ influence may not necessarily observe an equal influence on the solute binding strength. For instance, the atomic sites that lie along the $\Sigma 3$ GB plane and the $\{110\}$ planes further away from the TJ exhibit a small energetic preference, but the solute binding strength along these planes noticeably increases upon approaching the TJ. In the case of the $\Sigma 3-\Sigma 3-\Sigma 9$ TJ (Figures 9a-d), it was found that the TJ influence on solute binding behavior extended furthest along the $\Sigma 9 \mathrm{~GB}$, followed by a nearly symmetric influence along the $\Sigma 3$ GBs. This behavior can be explained on the basis of the higher interface energy/excess volume of the $\Sigma 9 \mathrm{~GB}$ as compared to the $\Sigma 3 \mathrm{GBs}$; whereby, the $\Sigma 9 \mathrm{~GB}$ offers a lower resistance path for the release of the strain energy due to the formation of the TJ. Furthermore, across the various solutes examined here, a sharp increase in the solute binding energy (almost twice the $\Sigma 9$ mean binding energy) was observed for sites along the $\Sigma 9 \mathrm{~GB}$ within a distance of 4-5 $\AA$ from the TJ (Figure 9a-d). For the $\Sigma 3-\Sigma 3-\Sigma 9 \mathrm{TJ}$, the largest region of TJ influence was observed for the interstitial hydrogen atom, this can be explained on the basis of the small atomic size of the hydrogen atom and the partially filled $s$ orbital enabling the preferential binding across a large selection of sites around the TJ. The size of the region over which the $\Sigma 3-\Sigma 3-\Sigma 9 \mathrm{TJ}$ exerts influence on solute binding behavior was found to be inversely related to the atomic size of the solute atom $(\mathrm{H}<\mathrm{He}<\mathrm{P}<\mathrm{V})$. However, in the case of the $\Sigma 3-\Sigma 11-\Sigma 33 \mathrm{TJ}$ (Figure 9e-h), the TJ influence extended over a smaller region in comparison to the $\Sigma 3-\Sigma 3-\Sigma 9 \mathrm{TJ}$ (except for the $\mathrm{V}$ atom) and was found to be insensitive to the type of solute atom. 


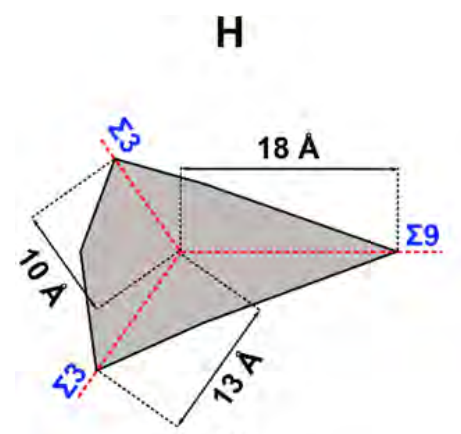

a)

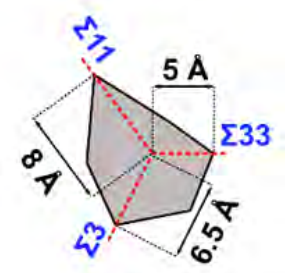

e)

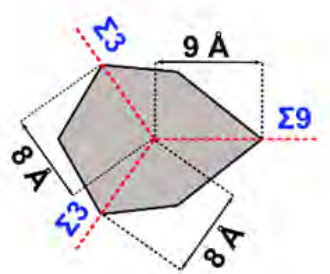

b)

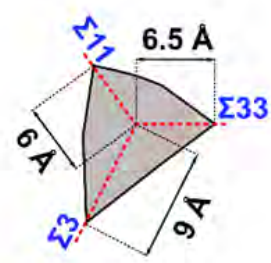

f)

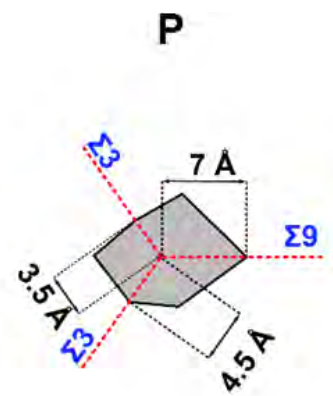

c)

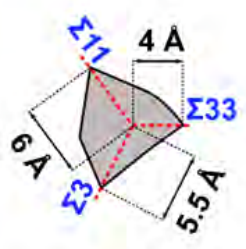

g)

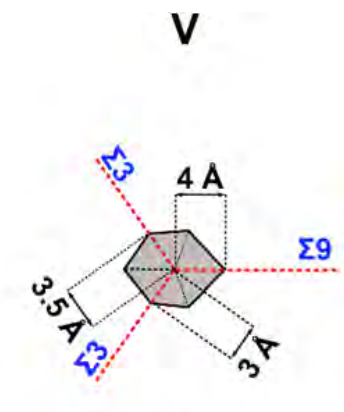

d)

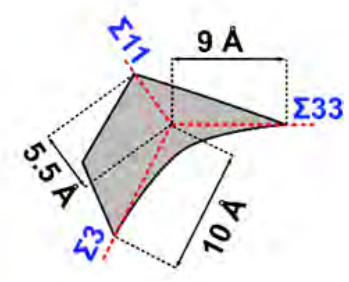

h)

Figure 9: A comparison of the length scale over which for (a-d) $\Sigma 3-\Sigma 3-\Sigma 9$ and (e-h) $\Sigma 3-\Sigma 11-\Sigma 33$ the probability of finding sites near the TJ with large binding energies for $\mathrm{H}, \mathrm{He}, \mathrm{P}$ and $\mathrm{V}$ atoms is high.

\section{Triple junction versus grain boundary: Solute segregation profile}

Previous studies $[42,87]$ have observed that the TJ demonstrates a greater tendency for the aggregation of solute atoms in a NC material as compared to the GBs. Therefore, using the solute binding behavior around the TJ quantified earlier, we compare the variation in solute concentration around the TJs and their constituent GBs using the Langmuir-McLean relationship [88] (refer to Figure 10), described in detail in Appendix B. To understand this behavior, we chose the $\Sigma 3-\Sigma 3-\Sigma 9 \mathrm{TJ}$ as it had the highest localization of TJ core energy (Figure $3 \mathrm{~b}$ and Table 2) and also showed the highest sink efficiency for the point defects and solute atoms examined here (Figure 8). Specifically, the concentration profile for $\mathrm{H}$ and $\mathrm{V}$ in the vicinity $(\leq 5 \AA)$ of the $\Sigma 3-\Sigma 3-\Sigma 9$ TJ were compared against the constituent GBs for a wide-range of temperature, see Figure 10. In the case of $\mathrm{H}$ (Figure 10a), the TJ had a 100\% sink efficiency up to a temperature of $500 \mathrm{~K}$. Beyond this temperature the sink efficiency decreases to $40 \%$ for a temperature of $1000 \mathrm{~K}$ (Figure 10a), indicating that at higher temperatures the solute atoms have sufficient activation energy available to diffuse away from the TJ. Further, the solute concentration within $\pm 10 \AA$ of the GB plane for the $\Sigma 3$ and $\Sigma 9$ GBs were examined individually with the help of the 
mean binding energy for the interstitial $\mathrm{H}$ atom [41]. The $\mathrm{H}$ concentration profiles for these constituent GBs were distinctly different when compared to the $\Sigma 3-\Sigma 3-\Sigma 9$ TJ. For instance, the ability of the $\Sigma 3 \mathrm{~GB}$ to act as a preferred site for aggregation of $\mathrm{H}$ atoms was found to diminish rapidly with increasing temperature (Figure 9a). Additionally, the $\Sigma 9$ GB showed a limited thermodynamic drive for the segregation of $\mathrm{H}$ along the GB.

In the case of a substitutional $\mathrm{V}$ atom, the concentration around the $\Sigma 3-\Sigma 3-\Sigma 9 \mathrm{TJ}$ drops sharply to almost $20 \%$ sink efficiency around room temperature (Figure $9 \mathrm{~b}$ ). Further, using the values for binding energy from the work of Rajagopalan et al. [60] it was found that the $\mathrm{V}$ atoms had close to $0 \%$ probability to accumulate along the constituent GBs across a wide-range of temperature (Figure 9b). These findings clearly demonstrate that despite limited potency of the constituent GBs, the TJs can have significantly higher sink efficiency for solute aggregation (Figure 10). Therefore, these atomic-scale insights provide the motivation for future studies examining the role of TJs on structural stability and sink efficiency which are key aspects in the engineering of radiation resistant $\mathrm{NC}$ materials.

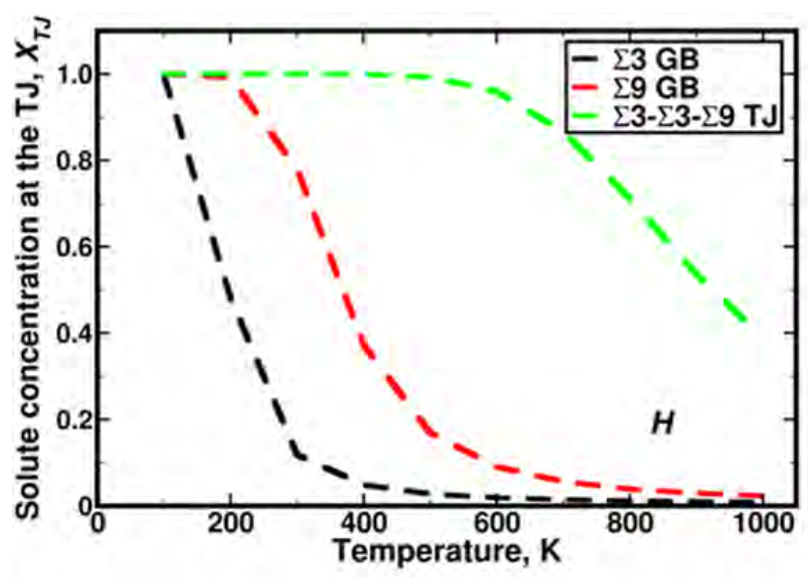

(a)

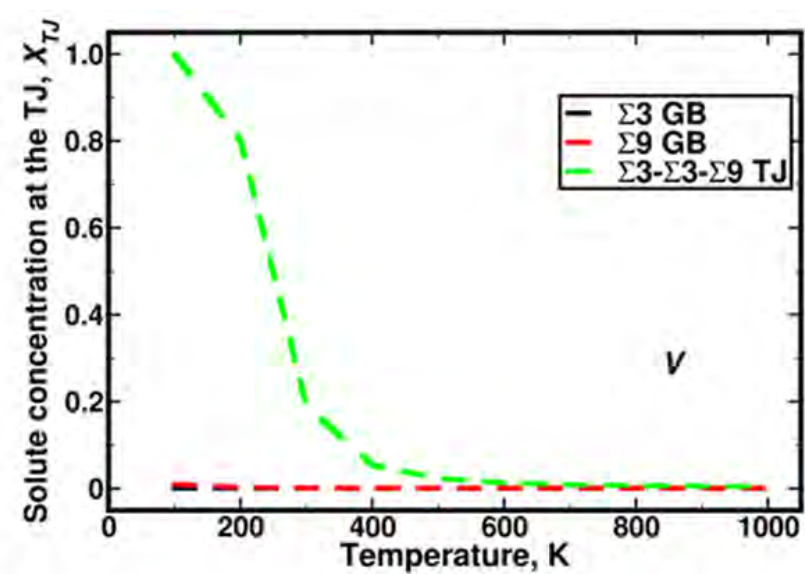

(b)

Figure 10: The distinct solute concentration in the vicinity of the $\mathrm{TJ}$ is highlighted by comparing the concentration profile across a wide range of temperature using Equation $\mathrm{S} 3\left(X_{b}^{H}=0.28 \%\right.$ and $X_{b}^{V}=0.08 \%$ ) for a) $\mathrm{H}$ and b) $\mathrm{V}$ in the vicinity of the $\Sigma 3-\Sigma 3-\Sigma 9 \mathrm{TJ}$ and its constituent GBs.

\section{Conclusions}

In this work, the role of TJs on the structural stability and the solute binding behavior was examined using atomistic simulations in $\alpha$-Fe. The key goals of this work were: a) systematically quantify the thermodynamic quantities at the $\mathrm{TJ}$ pertinent to the structural stability of $\mathrm{NC}$ 
material; b) examine the distinct role of the atomic arrangement surrounding the TJs on the point defect and solute atom binding behavior; c) measure the activation energy for vacancy diffusion along the various TJs and d) highlight the distinct role of the TJs on solute aggregation by comparing the concentration profile around the TJ and the constituent GBs using the LangmuirMcLean relationship [88]. The significant findings of this work are as follows:

1. The evolution of the TJ strain energy was found to clearly explain the point defect and solute atoms binding for all the TJs examined (refer Figures $3 b, 5$ and 7). For instance, the $\Sigma 3-\Sigma 3$ $\Sigma 9 \mathrm{TJ}$ had the highest TJ core energy, consequently both the point defects and solute atoms found several energetically stable binding sites in the immediate vicinity of the $\Sigma 3-\Sigma 3-\Sigma 9$ TJ.

2. The SIA was found to be energetically preferred over a vacancy defect in the vicinity of the TJs (Figure 4 and 5). Furthermore, the length scale over which the TJ exerted an influence on the binding behavior of SIA was much larger when compared to the vacancy binding behavior (Figure 4 and 5). However, for both the SIA and vacancy, a greater driving force to bind near the TJ was observed when compared to the constituent GBs. This clearly highlights the role of $\mathrm{TJ}$ as a strong sink for the accumulation of point defects that are formed by radiation damage. Understanding this is a key aspect in the engineering of radiation resistant $\mathrm{NC}$ material.

3. To comprehend the ramifications of the vacancy formation/binding behavior on the mass transport properties of NC material, the variation in the energy barrier for vacancy migration along the TJs was quantified using NEB calculations (Figure 6). The mean activation energy for vacancy diffusion along the TJ was found to have a strong inverse dependence on the resolved line tension measured at the various TJs. In other words, a TJ that had a large resolved line tension due to the intersection of GBs is thermodynamically less stable and was consequently found to result in a lower activation barrier for vacancy diffusion.

4. Finally, with the help of the Langmuir McLean relationship, we compared the solute concentration profiles for the $\Sigma 3-\Sigma 3-\Sigma 9 \mathrm{TJ}$ and its constituent GBs and it was found that the solute concentrations in the vicinity of the TJ can be much higher than the GBs (Figure 10). Physically, understanding solute concentration in the NC materials around the TJ can help in effectively decreasing the probability of crack nucleation around the TJ due to the embrittlement effect of solute atoms. 
In summary, it can be concluded that the physical properties exhibited at the TJ can be remarkably different from the constituent GBs. For instance, the $\Sigma 3-\Sigma 3-\Sigma 9 \mathrm{TJ}$ is formed by the intersection of two low energy $\Sigma 3$ GBs but has the highest localization of TJ strain energy (Figure $3 b$ ). This leads to the presence of energetically stable sites for the aggregation of point defects and solute atoms around the $\Sigma 3-\Sigma 3-\Sigma 9 \mathrm{TJ}$. Interestingly, our findings reveal that the $\Sigma 3$ $\Sigma 3-\Sigma 9$ and $\Sigma 3-\Sigma 27-\Sigma 81$ TJs had a high energy barrier for self-diffusion (Figure 7). This makes them an ideal candidate for tailoring radiation resistant microstructures, as they can effectively retard grain growth during irradiation. Atomistic studies are inherently limited to the finite length and time scales; therefore, a future study with a higher length scale modelling tool (e.g. phase field modeling) is necessary to further comprehend the TJ role on the microstructural stability. Nevertheless, our findings can guide future synergistic efforts that combine modelling and experimental approaches (such as the work by Beyerlein et al. [89-92] on bulk nanocomposite material) to advance the development of thermodynamically stable $\mathrm{NC}$ materials for nuclear applications.

\section{Acknowledgement}

The authors gratefully acknowledge support from the Office of Naval Research under contract N000141110793. 


\section{Appendix A. Quantifying thermodynamic properties for the TJ}

The net change in volume due to the formation of a TJ $\left(\Delta V_{T J}\right)$ was computed using the following relation:

$$
\begin{aligned}
& \Delta V_{T J}=\pi r_{T J}^{2}-\frac{n \omega_{\text {bulk }}}{r_{T J}}-\sum_{i=1}^{3} l_{x}^{i} \Delta V_{G B}^{i} \\
& r_{T J}=\max \left(l_{x}^{i}\right)
\end{aligned}
$$

where, $r_{T J}$ is the radius for the TJ assumed to be the maximum length of the unit vector tangential to the GBs intersecting at the TJ $\left(l_{x}^{i}\right), n$ is the number of atoms lying within a distance of $r_{T J}$ from the TJ, $\omega_{b u l k}$ is the atomic volume in a pristine BCC lattice at $0 \mathrm{~K}$, and $\Delta V_{G B}^{i}$ is the volume expansion along the GB plane per unit of the GB area for the $i^{\text {th }}$ interface. This expression for the $\Delta V_{T J}$ is indirectly related to the amount of misfit strain induced during formation of the TJ [93]. Figure Ala shows the net change in volume which was found to clearly increase with an increase in the $\Sigma$ value of the GBs involved. This suggests that the high $\Sigma$ GBs would form a more diffused TJ. In general, the volume change for TJs was found to follow an exponential relationship. Similar trends have been observed in previous studies on TJs in Si [93] and FCC materials [22].

The total excess energy due to the formation of the TJ was computed with respect to the energy of the pristine $\mathrm{BCC}$ lattice using the following relation:

$$
\Delta E_{T J}=\left(E_{t j}-n E_{c o h}-\sum_{i=1}^{3} E_{G B}^{i} l_{x}^{i} l_{z}\right) / l_{z}
$$

where, $E_{t j}$ is the energy of the defected atoms within the TJ and GBs, $E_{c o h}$ is the cohesive energy of a single $\alpha-\mathrm{Fe}$ atom at $0 \mathrm{~K}$, and $E_{G B}^{i}$ is the GB formation energy per unit area for the $i^{\text {th }}$ interface. The net excess energy was normalized by the line length $\left(l_{z}\right)$ of the TJ. These results were found by the summation of atomic energies within a distance of $190 \AA$ from the TJ. Figure A1b shows the net excess energy of the various TJs in $\alpha$-Fe. The net excess energy is found to be maximum at the $\Sigma 3-\Sigma 9-\Sigma 27 \mathrm{TJ}\left(5.25 \times 10^{-8} \mathrm{~J} / \mathrm{m}\right)$ and minimum at the $\Sigma 3-\Sigma 3-\Sigma 9 \mathrm{TJ}\left(3 \times 10^{-8} \mathrm{~J} / \mathrm{m}\right)$. For a few TJs studied in the open literature using atomistic methods $[44,93,94]$ and experimental work [95], the excess energies computed here were found to be in close agreement. This computed excess energy is the measure of additional elastic work required to bring together the three interfaces. Therefore, the excess energy directly provides a sense of the free energy that needs to be decreased either by the migration of the $\mathrm{TJ}$ or the emission of vacancies into the bulk. 


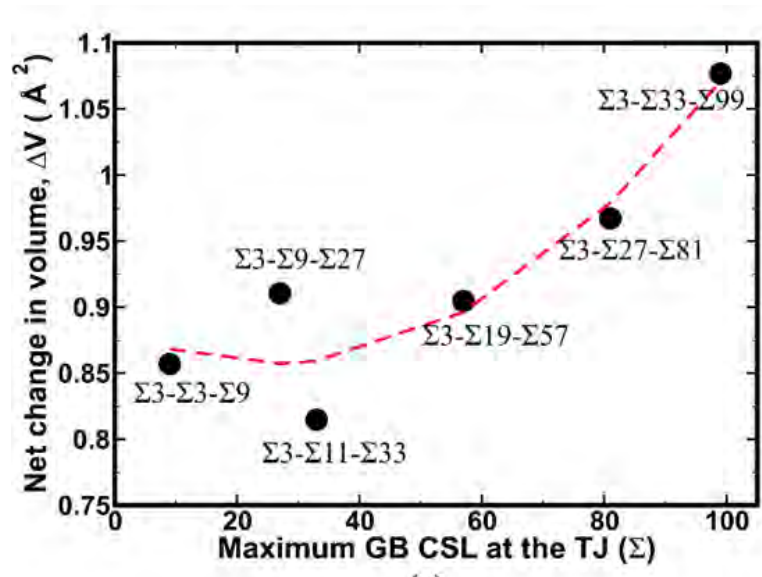

(a)

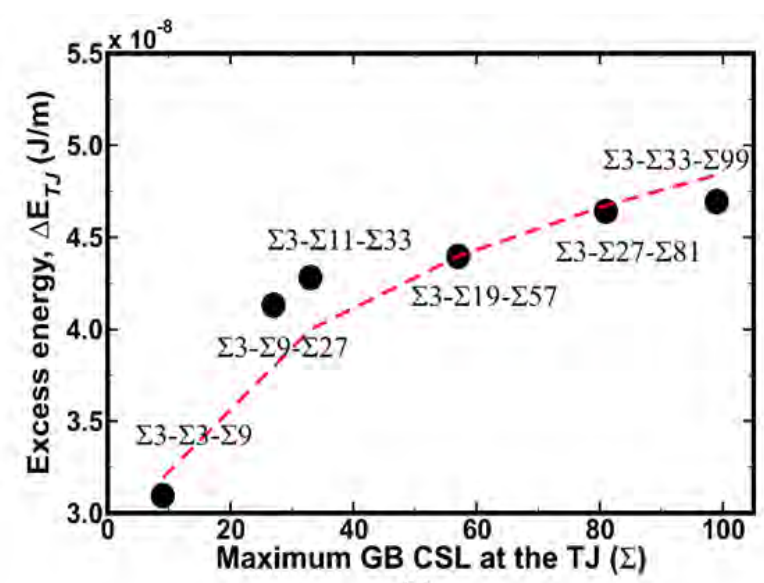

(b)

Figure A1: (a) The net change in volume at the TJ. In general, a clear trend can be observed that high CSL GB TJs underwent large volume change during formation of the TJ. (b) The excess energy due to the formation of the TJ.

\section{Appendix B. Solute concentration profile around the triple junction}

To gauge the physical ramifications of the atomic arrangement surrounding the TJs on the binding behavior for solute atoms, the Langmuir-McLean [88] relation was examined for the various TJs. Here, the normalized solute concentration at the $\mathrm{TJ}\left(X_{T J}\right)$ can be expressed in terms of the nominal bulk concentration of the solute $\left(X_{b}\right)$, the binding energy for various solute atoms in the vicinity of the $\mathrm{TJ}\left(E_{b}\right)$ and the temperature $(T)$ in the following manner:

$$
X_{T J}=1 /\left[1+\left(\frac{1-X_{b}}{X_{b}}\right) \exp \left(-E_{b} / k_{B} T\right)\right]
$$

where, $k_{B}$ is the Boltzmann constant. The mean binding energy $\left(\bar{E}_{b}\right)$ for all the atomic sites that lie within a radius of $5 \AA$ from the TJ was used in the Langmuir-Mclean relation (Equation S3) as the binding energy $\left(E_{b}\right)$. A temperature of $300 \mathrm{~K}(T)$ was chosen to examine the concentration profile for solutes surrounding the various TJ (Equation B1). In the case of $\mathrm{H}$ and $\mathrm{He}$ atoms, it was found that the vast majority $(\approx 100 \%)$ of the nominal bulk concentration $\left(X_{b}^{H}=0.28 \%\right.$ and $X_{b}^{H e}=0.3 \%$ ) preferred to reside around the various TJs examined here (Figure B1). In the case of the substitutional P atom, the concentration profile around the TJ (refer Figure B1) was found to be directly related to the magnitude of hydrostatic stress localization at the TJ (Figure 2). To elaborate, the $\Sigma 3-\Sigma 3-\Sigma 9\left(\sigma_{m}=3.78 \mathrm{GPa}\right), \Sigma 3-\Sigma 11-\Sigma 33\left(\sigma_{m}=5.20 \mathrm{GPa}\right)$ and $\Sigma 3-\Sigma 27-\Sigma 81\left(\sigma_{m}=2.05\right.$ GPa) TJs had large stress localization $(<5 \AA$ ) compared to the other TJs (Figure 2), thereby 
acting as the driving force for high concentrations of $\mathrm{P}\left(\approx 100 \%\right.$ of the $\left.X_{b}^{P}=0.1 \%\right)$ around these TJs. However, in the case of $\mathrm{V}$ there was a limited tendency for localization of high concentrations around the TJs (Figure B1). This observation can be attributed to the large size of the substitutional $\mathrm{V}$ atom compared to the $\mathrm{P}$ atom $\left(r_{V} \sim 1.4 r_{P}\right)$, thereby decreasing the probability of finding large concentrations of $\mathrm{V}$ in the vicinity of the TJs.

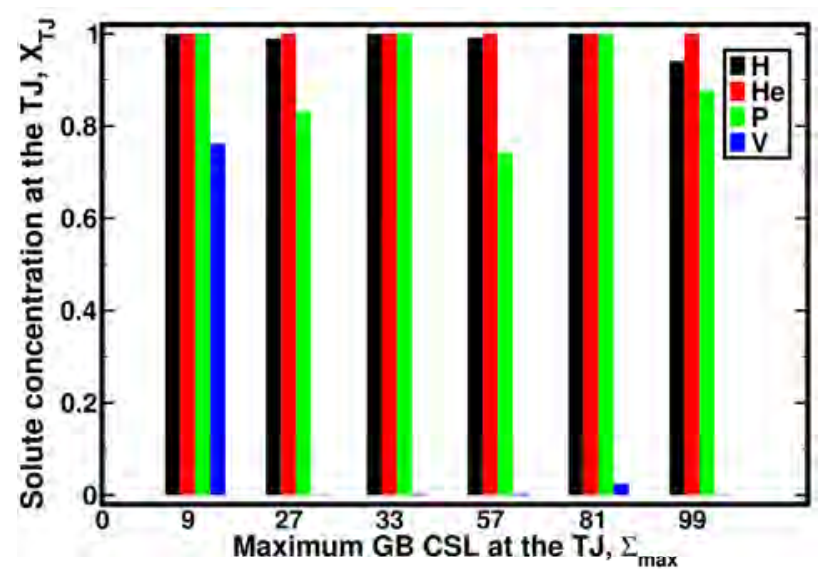

Figure B1: The solute concentration for the various TJs in terms of nominal solute concentration of the solute in the bulk $\left(X_{b}^{H}=0.28 \%, X_{b}^{H e}=0.3 \%, X_{b}^{P}=0.1 \%\right.$ and $\left.X_{b}^{V}=0.08 \%\right)$ at $300 \mathrm{~K}$. 


\section{References}

[1] G.R. Odette, M.J. Alinger, B.D. Wirth, Recent developments in irradiation-resistant steels, Annu Rev Mater Res. 38 (2008) 471-503.

[2] I.J. Beyerlein, A. Caro, M.J. Demkowicz, N.A. Mara, A. Misra, B.P. Uberuaga, Radiation damage tolerant nanomaterials, Mater. Today. 16 (2013) 443-449. doi:10.1016/j.mattod.2013.10.019.

[3] S.J. Zinkle, G.S. Was, Materials challenges in nuclear energy, Acta Mater. 61 (2013) 735758. doi:10.1016/j.actamat.2012.11.004.

[4] B.N. Singh, T. Leffers, W.V. Green, M. Victoria, Grain boundary related effects in aluminium during $600 \mathrm{MeV}$ proton irradiation at different temperatures, J. Nucl. Mater. 122 (1984) 703-708.

[5] S.J. Zinkle, K. Farrell, Void swelling and defect cluster formation in reactor-irradiated copper, J. Nucl. Mater. 168 (1989) 262-267.

[6] L.S. Novikov, V.N. Mileev, E.N. Voronina, L.I. Galanina, A.A. Makletsov, V.V. Sinolits, Radiation effects on spacecraft materials, J. Surf. Investig. X-Ray Synchrotron Neutron Tech. 3 (2009) 199-214.

[7] E.M. Bringa, J.D. Monk, A. Caro, A. Misra, L. Zepeda-Ruiz, M. Duchaineau, F. Abraham, M. Nastasi, S.T. Picraux, Y.Q. Wang, others, Are nanoporous materials radiation resistant?, Nano Lett. 12 (2011) 3351-3355.

[8] M. Rose, A.G. Balogh, H. Hahn, Instability of irradiation induced defects in nanostructured materials, Nucl. Instrum. Methods Phys. Res. Sect. B Beam Interact. Mater. At. 127-128 (1997) 119-122. doi:10.1016/S0168-583X(96)00863-4.

[9] X.-M. Bai, A.F. Voter, R.G. Hoagland, M. Nastasi, B.P. Uberuaga, Efficient Annealing of Radiation Damage Near Grain Boundaries via Interstitial Emission, Science. 327 (2010) 1631-1634. doi:10.1126/science.1183723.

[10] K.Y. Yu, Y. Liu, C. Sun, H. Wang, L. Shao, E.G. Fu, X. Zhang, Radiation damage in helium ion irradiated nanocrystalline Fe, J. Nucl. Mater. 425 (2012) 140-146. doi:10.1016/j.jnucmat.2011.10.052.

[11] B.C. Hornbuckle, T. Rojhirunsakool, M. Rajagopalan, T. Alam, G.P.P. Pun, R. Banerjee, K.N. Solanki, Y. Mishin, L.J. Kecskes, K.A. Darling, Effect of Ta Solute Concentration on the Microstructural Evolution in Immiscible Cu-Ta Alloys, JOM. 67 (2015) 2802-2809. doi:10.1007/s11837-015-1643-x.

[12] M. Bhatia, M. Rajagopalan, K. Darling, M. Tschopp, K. Solanki, The role of Ta on twinnability in nanocrystalline $\mathrm{Cu}-\mathrm{Ta}$ alloys, Mater. Res. Lett. (2016). doi: $10.1080 / 21663831.2016 .1201160$.

[13] A. Misra, M.J. Demkowicz, X. Zhang, R.G. Hoagland, The radiation damage tolerance of ultra-high strength nanolayered composites, Jom. 59 (2007) 62-65.

[14] X. Zhang, N. Li, O. Anderoglu, H. Wang, J.G. Swadener, T. Höchbauer, A. Misra, R.G. Hoagland, Nanostructured $\mathrm{Cu} / \mathrm{Nb}$ multilayers subjected to helium ion-irradiation, Nucl. Instrum. Methods Phys. Res. Sect. B Beam Interact. Mater. At. 261 (2007) 1129-1132.

[15] A.M. Ibrahim, Analysis of grain growth due to ion irradiation of thin films, Nucl. Instrum. Methods Phys. Res. Sect. B Beam Interact. Mater. At. 29 (1988) 650-652.

[16] N. Karpe, J. Bøttiger, N.G. Chechenin, J.P. Krog, Proceedings of the Eighth International Conference on Rapidly Quenched and Metastable Materials Ion irradiation induced grain 
growth in nanocrystalline $\mathrm{Fe}$ and Fe (Zr), Mater. Sci. Eng. A. 179 (1994) 582-586. doi:10.1016/0921-5093(94)90271-2.

[17] N. Nita, R. Schaeublin, M. Victoria, R.Z. Valiev, Effects of irradiation on the microstructure and mechanical properties of nanostructured materials, Philos. Mag. 85 (2005) 723-735. doi:10.1080/14786430412331319965.

[18] D.C. Bufford, F.F. Abdeljawad, S.M. Foiles, K. Hattar, Unraveling irradiation induced grain growth with in situ transmission electron microscopy and coordinated modeling, Appl. Phys. Lett. 107 (2015) 191901. doi:10.1063/1.4935238.

[19] W. Mohamed, B. Miller, D. Porter, K. Murty, The Role of Grain Size on Neutron Irradiation Response of Nanocrystalline Copper, Materials. 9 (2016) 144. doi:10.3390/ma9030144.

[20] H. Gleiter, Nanocrystalline materials, Prog. Mater. Sci. 33 (1989) 223-315. doi:10.1016/0079-6425(89)90001-7.

[21] H. Gleiter, Nanostructured materials: basic concepts and microstructure, Acta Mater. 48 (2000) 1-29. doi:10.1016/S1359-6454(99)00285-2.

[22] I. Adlakha, K.N. Solanki, Structural stability and energetics of grain boundary triple junctions in face centered cubic materials, Sci. Rep. 5 (2015). doi:10.1038/srep08692.

[23] M.A. Meyers, A. Mishra, D.J. Benson, Mechanical properties of nanocrystalline materials, Prog. Mater. Sci. 51 (2006) 427-556.

[24] P.R. Okamoto, H. Wiedersich, Segregation of alloying elements to free surfaces during irradiation, J. Nucl. Mater. 53 (1974) 336-345.

[25] R.A. Johnson, N.Q. Lam, Solute segregation in metals under irradiation, Phys. Rev. B. 13 (1976) 4364-4375. doi:10.1103/PhysRevB.13.4364.

[26] N.Q. Lam, P.R. Okamoto, R.A. Johnson, Solute segregation and precipitation under heavyion bombardment, J. Nucl. Mater. 78 (1978) 408-418. doi:10.1016/0022-3115(78)90462-2.

[27] H. Ullmaier, The influence of helium on the bulk properties of fusion reactor structural materials, Nucl. Fusion. 24 (1984) 1039.

[28] R.E. Stoller, The influence of helium on microstructural evolution: Implications for DT fusion reactors, J. Nucl. Mater. 174 (1990) 289-310.

[29] E.E. Bloom, J.T. Busby, C.E. Duty, P.J. Maziasz, T.E. McGreevy, B.E. Nelson, B.A. Pint, P.F. Tortorelli, S.J. Zinkle, Critical questions in materials science and engineering for successful development of fusion power, J. Nucl. Mater. 367 (2007) 1-10.

[30] T. Diaz de la Rubia, H.M. Zbib, T.A. Khraishi, B.D. Wirth, M. Victoria, M.J. Caturla, Multiscale modelling of plastic flow localization in irradiated materials, Nature. 406 (2000) 871-874. doi:10.1038/35022544.

[31] A. Möslang, T. Wiss, Materials for energy: From fission towards fusion, Nat. Mater. 5 (2006) 679-680. doi:10.1038/nmat1715.

[32] W.T. Geng, A.J. Freeman, R. Wu, G.B. Olson, Effect of Mo and Pd on the grain-boundary cohesion of Fe, Phys. Rev. B. 62 (2000) 6208-6214. doi:10.1103/PhysRevB.62.6208.

[33] J.P. Buban, K. Matsunaga, J. Chen, N. Shibata, W.Y. Ching, T. Yamamoto, Y. Ikuhara, Grain Boundary Strengthening in Alumina by Rare Earth Impurities, Science. 311 (2006) 212-215. doi:10.1126/science.1119839.

[34] M. Yamaguchi, First-Principles Study on the Grain Boundary Embrittlement of Metals by Solute Segregation: Part I. Iron (Fe)-Solute (B, C, P, and S) Systems, Metall. Mater. Trans. A. 42 (2011) 319-329. doi:10.1007/s11661-010-0381-5. 
[35] C.J. McMahon Jr., V. Vitek, The effects of segregated impurities on intergranular fracture energy, Acta Metall. 27 (1979) 507-513. doi:10.1016/0001-6160(79)90002-6.

[36] D.N. Seidman, B.W. Krakauer, D. Udler, Atomic scale studies of solute-atom segregation at grain boundaries: Experiments and simulations, J. Phys. Chem. Solids. 55 (1994) 10351057. doi:10.1016/0022-3697(94)90123-6.

[37] A. Sutton, R. Balluffi, Interfaces in Crystalline Materials, Clarendon Press, 1995.

[38] E.D. Hondros, M.P. Seah, Segregation to interfaces, Int. Met. Rev. 22 (1977) 262-301.

[39] P. Lejček, S. Hofmann, Thermodynamics and structural aspects of grain boundary segregation, Crit. Rev. Solid State Mater. Sci. 20 (1995) 1-85. doi:10.1080/10408439508243544.

[40] M. Yamaguchi, M. Shiga, H. Kaburaki, Grain Boundary Decohesion by Impurity Segregation in a Nickel-Sulfur System, Science. 307 (2005) 393-397. doi:10.1126/science.1104624.

[41] K.N. Solanki, M.A. Tschopp, M.A. Bhatia, N.R. Rhodes, Atomistic Investigation of the Role of Grain Boundary Structure on Hydrogen Segregation and Embrittlement in $\alpha$-Fe, Metall. Mater. Trans. A. 44 (2013) 1365-1375. doi:10.1007/s11661-012-1430-z.

[42] K.-M. Yin, A. h. King, T. e. Hsieh, F.-R. Chen, J. j. Kai, L. Chang, Segregation of Bismuth to Triple Junctions in Copper, Microsc. Microanal. 3 (1997) 417-422. doi:10.1017/S1431927697970318.

[43] G. Henkelman, B.P. Uberuaga, H. Jónsson, A climbing image nudged elastic band method for finding saddle points and minimum energy paths, J. Chem. Phys. 113 (2000) 99019904.

[44] P. Fortier, G. Palumbo, G.D. Bruce, W.A. Miller, K.T. Aust, Triple line energy determination by scanning tunneling microscopy, Scr. Metall. Mater. 25 (1991) 177-182. doi:10.1016/0956-716X(91)90376-C.

[45] P. Fortier, W.A. Miller, K.T. Aust, Triple junction and grain boundary character distributions in metallic materials, Acta Mater. 45 (1997) 3459-3467. doi:10.1016/S13596454(97)00004-9.

[46] G.S. Rohrer, E.A. Holm, A.D. Rollett, S.M. Foiles, J. Li, D.L. Olmsted, Comparing calculated and measured grain boundary energies in nickel, Acta Mater. 58 (2010) 50635069.

[47] S. Plimpton, Fast parallel algorithms for short-range molecular dynamics, J. Comput. Phys. 117 (1995) 1-19.

[48] G.J. Ackland, K. D’Mellow, S.L. Daraszewicz, D.J. Hepburn, M. Uhrin, K. Stratford, The MOLDY short-range molecular dynamics package, Comput. Phys. Commun. 182 (2011) 2587-2604.

[49] A. Ramasubramaniam, M. Itakura, E.A. Carter, Interatomic potentials for hydrogen in $\alpha-$ iron based on density functional theory, Phys. Rev. B. 79 (2009) 174101.

[50] F. Gao, H. Deng, H.L. Heinisch, R.J. Kurtz, A new Fe-He interatomic potential based on $<$ i $>$ ab initio $</ \mathrm{i}>$ calculations in $\alpha$-Fe, J. Nucl. Mater. 418 (2011) 115-120.

[51] G.J. Ackland, M.I. Mendelev, D.J. Srolovitz, S. Han, A.V. Barashev, Development of an interatomic potential for phosphorus impurities in $\alpha$-iron, J. Phys. Condens. Matter. 16 (2004) S2629. doi:10.1088/0953-8984/16/27/003.

[52] M.I. Mendelev, S. Han, W. Son, G.J. Ackland, D.J. Srolovitz, Simulation of the interaction between Fe impurities and point defects in V, Phys. Rev. B. 76 (2007) 214105. 
[53] M.I. Mendelev, S. Han, D.J. Srolovitz, G.J. Ackland, D.Y. Sun, M. Asta, Development of new interatomic potentials appropriate for crystalline and liquid iron, Philos. Mag. 83 (2003) 3977-3994.

[54] H. Chamati, N.I. Papanicolaou, Y. Mishin, D.A. Papaconstantopoulos, Embedded-atom potential for $\mathrm{Fe}$ and its application to self-diffusion on Fe(1 0 0), Surf. Sci. 600 (2006) 1793-1803. doi:10.1016/j.susc.2006.02.010.

[55] K. Refson, Moldy: a portable molecular dynamics simulation program for serial and parallel computers, Comput. Phys. Commun. 126 (2000) 310-329. doi:10.1016/S00104655(99)00496-8.

[56] F. Gao, H. Deng, H.L. Heinisch, R.J. Kurtz, A new Fe-He interatomic potential based on $\mathrm{ab}$ initio calculations in $\alpha$-Fe, J. Nucl. Mater. 418 (2011) 115-120. doi:10.1016/j.jnucmat.2011.06.008.

[57] M.I. Mendelev, S. Han, W. Son, G.J. Ackland, D.J. Srolovitz, Simulation of the interaction between Fe impurities and point defects in V, Phys. Rev. B. 76 (2007) 214105. doi:10.1103/PhysRevB.76.214105.

[58] G.J. Ackland, M.I. Mendelev, D.J. Srolovitz, S. Han, A.V. Barashev, Development of an interatomic potential for phosphorus impurities in $\alpha$-iron, J. Phys. Condens. Matter. 16 (2004) S2629. doi:10.1088/0953-8984/16/27/003.

[59] M.A. Tschopp, F. Gao, L. Yang, K.N. Solanki, Binding Energetics of Substitutional and Interstitial Helium and Di-Helium Defects with Grain Boundary Structure in alpha-Fe, ArXiv E-Prints. 1309.6337 (2013) 6337.

[60] M. Rajagopalan, M.A. Tschopp, K.N. Solanki, Grain boundary segregation of interstitial and substitutional impurity atoms in alpha-iron, JOM. 66 (2014) 129-138.

[61] M.A. Bhatia, S. Groh, K.N. Solanki, Atomic-scale investigation of point defects and hydrogen-solute atmospheres on the edge dislocation mobility in alpha iron, J. Appl. Phys. 116 (2014) 64302. doi:10.1063/1.4892630.

[62] R.C. Pond, V. Vitek, Periodic Grain Boundary Structures in Aluminium. I. A Combined Experimental and Theoretical Investigation of Coincidence Grain Boundary Structure in Aluminium, Proc. R. Soc. Lond. Ser. Math. Phys. Sci. 357 (1977) 453-470. doi: $10.2307 / 79397$.

[63] A. Sutton, On the structural unit model of grain boundary structure, Philos. Mag. Lett. 59 (1989) 53-59.

[64] M.A. Tschopp, K.N. Solanki, F. Gao, X. Sun, M.A. Khaleel, M.F. Horstemeyer, Probing grain boundary sink strength at the nanoscale: Energetics and length scales of vacancy and interstitial absorption by grain boundaries in $\alpha$-Fe, Phys. Rev. B. 85 (2012) 64108. doi:10.1103/PhysRevB.85.064108.

[65] M.A. Bhatia, K.N. Solanki, Energetics of vacancy segregation to symmetric tilt grain boundaries in hexagonal closed pack materials, J. Appl. Phys. 114 (2013) 244309. doi: $10.1063 / 1.4858401$.

[66] I. Adlakha, M.A. Bhatia, M.A. Tschopp, K.N. Solanki, Atomic scale investigation of grain boundary structure role on intergranular deformation in aluminium, Philos. Mag. 94 (2014) 3445-3466. doi:10.1080/14786435.2014.961585.

[67] I. Adlakha, M.A. Tschopp, K.N. Solanki, The role of grain boundary structure and crystal orientation on crack growth asymmetry in aluminum, Mater. Sci. Eng. A. 618 (2014) 345354. 
[68] I. Adlakha, K.N. Solanki, Critical assessment of hydrogen effects on the slip transmission across grain boundaries in $\alpha-F e$, Proc R Soc A. 472 (2016) 20150617. doi:10.1098/rspa.2015.0617.

[69] R. Kremer, R. Narayanan, S. Shekhar, A.H. King, On the design of controlled tricrystal specimens for the systematic investigation of static grain boundary triple junction properties, J. Mater. Sci. 40 (2005) 2795-2802.

[70] M.S. Wu, K. Zhou, A.A. Nazarov, Crack nucleation at disclinated triple junctions, Phys. Rev. B. 76 (2007) 134105.

[71] M. Rajagopalan, M.A. Bhatia, M.A. Tschopp, D.J. Srolovitz, K.N. Solanki, Atomic-scale analysis of liquid-gallium embrittlement of aluminum grain boundaries, Acta Mater. 73 (2014) 312-325. doi:10.1016/j.actamat.2014.04.011.

[72] M.A. Tschopp, F. Gao, K.N. Solanki, Binding of HenV clusters to $\alpha$-Fe grain boundaries, J. Appl. Phys. 115 (2014) 233501. doi:10.1063/1.4883357.

[73] D. Sheppard, R. Terrell, G. Henkelman, Optimization methods for finding minimum energy paths, J. Chem. Phys. 128 (2008) 134106. doi:10.1063/1.2841941.

[74] G. Gottstein, L.S. Shvindlerman, B. Zhao, Thermodynamics and kinetics of grain boundary triple junctions in metals: Recent developments, Scr. Mater. 62 (2010) 914-917.

[75] J.P. Hirth, J. Lothe, Theory of Dislocations, 2nd, Ed John Willey Sons. (1982).

[76] J.D. Eshelby, W.T. Read, W. Shockley, Anisotropic elasticity with applications to dislocation theory, Acta Metall. 1 (1953) 251-259. doi:10.1016/0001-6160(53)90099-6.

[77] A.J.E. Foreman, Dislocation energies in anisotropic crystals, Acta Metall. 3 (1955) 322330. doi:10.1016/0001-6160(55)90036-5.

[78] M.A. Tschopp, K. Solanki, F. Gao, X. Sun, M. Khaleel, M. Horstemeyer, Probing grain boundary sink strength at the nanoscale: Energetics and length scales of vacancy and interstitial absorption by grain boundaries in $\alpha$-Fe, Phys. Rev. B. 85 (2012). doi:10.1103/PhysRevB.85.064108.

[79] N. Nita, R. Schaeublin, M. Victoria, Impact of irradiation on the microstructure of nanocrystalline materials, J. Nucl. Mater. 329-333, Part B (2004) 953-957. doi:10.1016/j.jnucmat.2004.04.058.

[80] T.D. Shen, F. Shihai, T. Ming, J.A. Valdez, W. Yongqiang, K.E. Sickafus, Enhanced radiation tolerance in nanocrystalline MgGa $\$\{$ sub $2 \$\}$ O $\$\{$ sub 4\$ $\$$, Appl. Phys. Lett. 90 (2007). http://www.osti.gov/scitech/biblio/20971990 (accessed November 4, 2015).

[81] S. Kobayashi, S. Tsurekawa, T. Watanabe, Structure-dependent triple junction hardening and intergranular fracture in molybdenum, Philos. Mag. 86 (2006) 5419-5429. doi:10.1080/14786430600672711.

[82] G. Gottstein, A.H. King, L.S. Shvindlerman, The effect of triple-junction drag on grain growth, Acta Mater. 48 (2000) 397-403. doi:10.1016/S1359-6454(99)00373-0.

[83] A.H. King, Triple lines in materials science and engineering, Scr. Mater. 62 (2010) 889893. doi:10.1016/j.scriptamat.2010.02.020.

[84] G. Gottstein, Y. Ma, L.S. Shvindlerman, Triple junction motion and grain microstructure evolution, Acta Mater. 53 (2005) 1535-1544. doi:10.1016/j.actamat.2004.12.006.

[85] U. Czubayko, V.G. Sursaeva, G. Gottstein, L.S. Shvindlerman, Influence of triple junctions on grain boundary motion, Acta Mater. 46 (1998) 5863-5871. doi:10.1016/S13596454(98)00241-9.

[86] V.V. Zabil'skii, Temper embrittlement of structural alloy steels (review), Met. Sci. Heat Treat. 29 (1987) 32-42. doi:10.1007/BF00735489. 
[87] P. Stender, Z. Balogh, G. Schmitz, Triple junction segregation in nanocrystalline multilayers, Phys. Rev. B. 83 (2011) 121407. doi:10.1103/PhysRevB.83.121407.

[88] D. McLean, Grain Boundary Segregation in Metals, (1957).

[89] M.J. Demkowicz, P. Bellon, B.D. Wirth, Atomic-scale design of radiation-tolerant nanocomposites, MRS Bull. 35 (2010) 992-998.

[90] I.J. Beyerlein, J. Wang, R. Zhang, Interface-dependent nucleation in nanostructured layered composites, APL Mater. 1 (2013) 32112.

[91] N.A. Mara, I.J. Beyerlein, Interface-dominant multilayers fabricated by severe plastic deformation: Stability under extreme conditions, Curr. Opin. Solid State Mater. Sci. 19 (2015) 265-276.

[92] E. Martínez, A. Caro, I.J. Beyerlein, Atomistic modeling of defect-induced plasticity in CuNb nanocomposites, Phys. Rev. B. 90 (2014) 54103. doi:10.1103/PhysRevB.90.054103.

[93] S. Costantini, P. Alippi, L. Colombo, F. Cleri, Triple junctions and elastic stability of polycrystalline silicon, Phys. Rev. B. 63 (2000) 45302.

[94] S. Shekhar, A.H. King, Strain fields and energies of grain boundary triple junctions, Acta Mater. 56 (2008) 5728-5736. doi:10.1016/j.actamat.2008.07.053.

[95] S.G. Protasova, V.G. Sursaeva, L.S. Shvindlerman, Study of the motion of individual triple junctions in aluminum, Phys. Solid State. 45 (2003) 1471-1474. 


\section{Graphical Abstract}

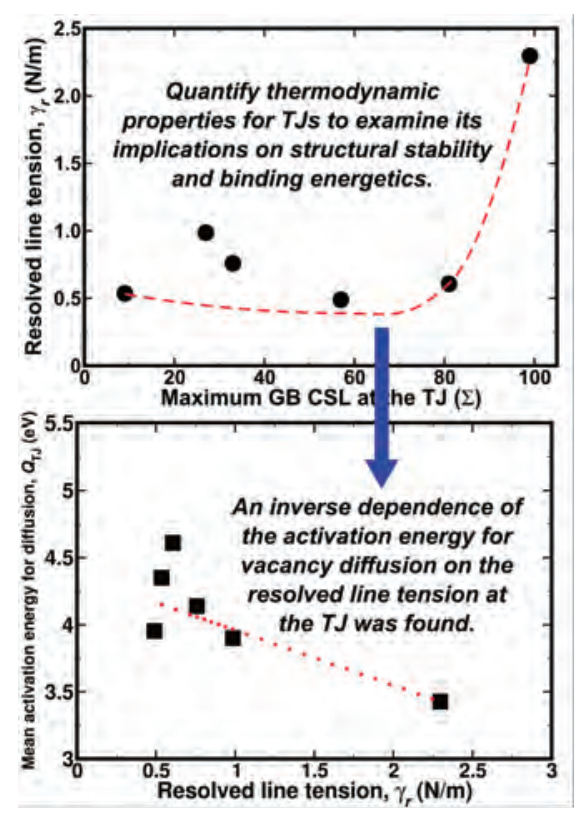

Role of point defects on structural stability and energetics of grain boundary triple junctions in $\alpha-F e$

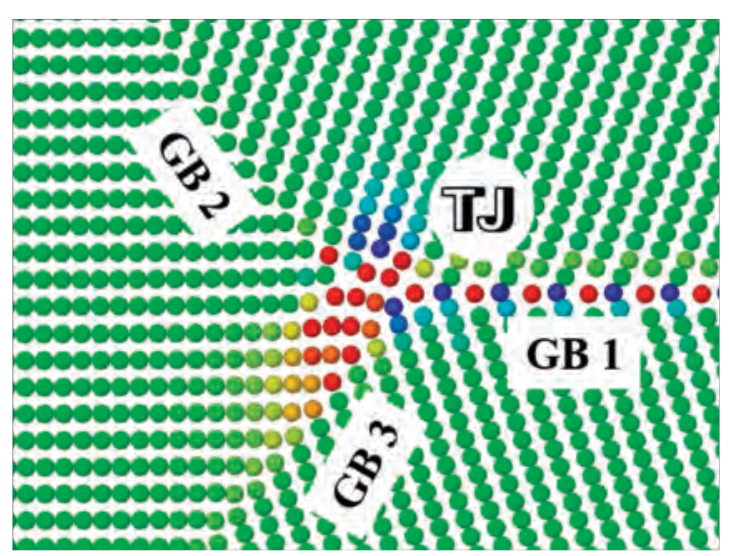

These findings can guide future efforts to advance the development of thermodynamically stable NC materials for nuclear applications.

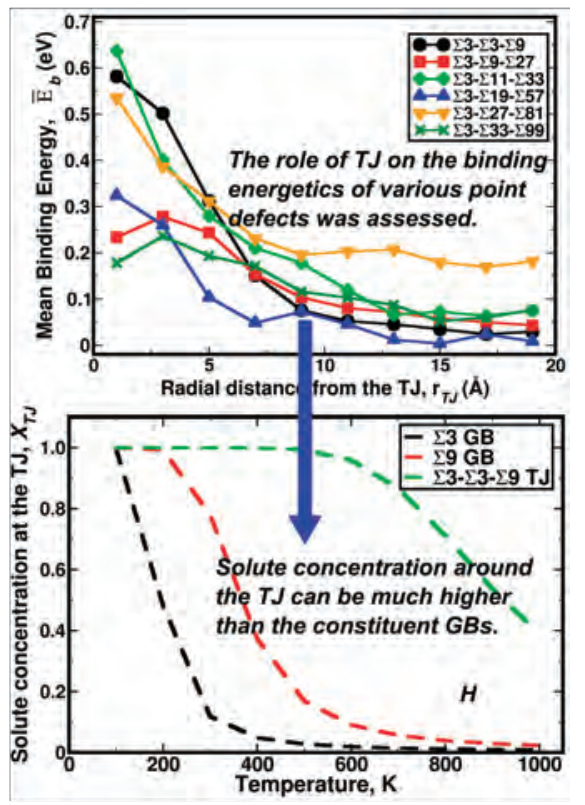

